\title{
SEXTACTIC POINTS ON A SIMPLE CLOSED CURVE
}

\author{
GUDLAUGUR THORBERGSSON AND MASAAKI UMEHARA
}

\begin{abstract}
We give optimal lower bounds for the number of sextactic points on a simple closed curve in the real projective plane. Sextactic points are after inflection points the simplest projectively invariant singularities on such curves. Our method is axiomatic and can be applied in other situations.
\end{abstract}

\section{$\S 1$. Introduction}

In analogy with tangent lines and inflection points of regular curves in the real or complex projective plane, one can consider their osculating conics and sextactic points. Choose five points on a curve $\gamma$ in a neighborhood of a point $p$ on $\gamma$ that is not an inflection point. There is a unique regular conic passing through the five points. Letting the five points all converge to $p$, the conics converge to a uniquely defined regular conic that is called the osculating conic of $\gamma$ in $p$. The osculating conic meets $\gamma$ with multiplicity at least five in $p$. If it meets with multiplicity at least six in $p$, then $p$ is called a sextactic point.

Inflection and sextactic points on curves in the complex projective plane were well understood already in the nineteenth century. We will make some historic remarks on this towards the end of the introduction. It is the case of curves in the real projective plane that still poses problems.

In the present paper we will be dealing with closed $C^{\infty}$-parameterized curves $\gamma: S^{1} \rightarrow P^{2}$ that are simple (free of self-intersections) and regular (nowhere vanishing tangent vector). Here and elsewhere in the paper we let $P^{2}$ denote the real projective plane. The existence of inflection and sextactic points on such curves has of course been much studied. Of importance for us is the result of Möbius [Mö] that a simple regular curve in $P^{2}$ that is not null-homotopic has at least three inflection points. As far as we know,

Received December 25, 2000.

1991 Mathematics Subject Classification: Primary 53A15, Secondary 51L15, 53C75, $53 \mathrm{~A} 25$. 
the first paper to deal with sextactic points on curves in the real projective (or affine) plane that are not necessarily algebraic, is the paper [Mu1] of Mukhopadhyaya from the year 1909. There it is proved that a strictly convex curve in the affine plane has at least six sextactic points. An independent proof of this theorem due to Herglotz and Radon was published by Blaschke [Bl1] in 1917. Proofs can also be found in the textbooks [Bl2] and [Bo]. In [Mu2] Mukhopadhyaya proved that three of these sextactic points can be chosen so that the corresponding osculating conics are inscribed and other three such that the corresponding osculating conics are circumscribed. We are not aware of any results on sextactic points on curves that are not strictly convex. For recent papers on sextactic points on strictly convex curves and related matters, see e.g. [Ar3] and [GMO].

Our main results are summarized in the following three theorems. We have not tried to state here everything in its strongest form. More precise results can be found in Sections 4 and 5 . Notice that we will give examples in Appendices $\mathrm{B}$ and $\mathrm{C}$ showing that all these theorems are optimal. Two of the examples in Appendix B were communicated to us by Izumiya and Sano [IS] who found them in their study of affine evolutes.

THEOREM 1.1. Let $\gamma$ be a simple closed curve in $P^{2}$ that is not nullhomotopic. Then $\gamma$ has at least three sextactic points.

The result of Möbius mentioned above is one of the essential ingredients in the proof of this theorem. Notice that the theorem is optimal since the noncontractible branch of a real cubic has exactly three sextactic points as we will explain in Appendix C. Notice also that the theorem was stated as a problem by $\mathrm{Bol}$ in [Bo] on p. 43. A sketch of a proof of Theorem 1.1 under rather strong genericity assumptions on the inflection points of $\gamma$ was given by Fabricius-Bjerre in [Fa]; see Remark (iii) after Proposition 5.1.

THEOREM 1.2. Let $\gamma$ be a simple closed curve in $P^{2}$ that is nullhomotopic.

(i) (Mukhopadhyaya) If $\gamma$ is strictly convex, then it has at least six sextactic points.

(ii) If $\gamma$ is not convex, then it has at least three sextactic points.

(iii) If $\gamma$ is convex, then it has at least two sextactic points. 
Part (i), or Mukhopadhyaya's theorem, is optimal, since a nullhomotopic component of a regular real cubic is strictly convex and has exactly six sextactic points as we will explain in Appendix C. That the other parts are optimal will be explained in Appendix B.

Counting sextactic points and inflection points together, we can prove the following theorem.

THEOREM 1.3. Let $\gamma$ be a simple closed curve in $P^{2}$ that is nullhomotopic. Then the total number of sextactic and inflection points on $\gamma$ is at least four.

This theorem is optimal as an example of Izumiya and Sano [IS] shows that we explain in Appendix B. It will be clear from the proof that Theorem 1.3 can only be optimal for convex curves with one or two inflection points.

The arguments in our proofs are inspired by those of Mukhopadhyaya in [Mu1] and especially in [Mu2], although there are of course new ideas needed to deal with curves with inflection points. We have chosen an axiomatic approach that is similar in spirit to the one introduced by the second author in $[\mathrm{Um}]$ to deal with vertices and was further studied in [TU1]. The main idea behind this approach was motivated by the paper [Kn] of H. Kneser. It should be pointed out that our theorems are more generally true for curves that are only $C^{4}$ with essentially the same proofs, see the remark after Proposition 5.1, but we stay in the $C^{\infty}$-category to simplify the exposition. Notice that one has to modify the definition of a sextactic point in the case of $C^{4}$-curves, see Section 2.

We would like to make a few remarks on inflection and sextactic points on algebraic curves in the complex projective plane. There is a formula due to Plücker (1835) that one can find in most textbooks on algebraic curves saying that a regular algebraic curve $\gamma$ of degree $d$ in $P^{2}(\mathbf{C})$ has exactly $3 d(d-2)$ inflection points counted with multiplicities. It is much less known that Cayley [Ca2] proved in 1865 that such a curve (with simple inflection points) has exactly $3 d(4 d-9)$ sextactic points counted with multiplicities. The condition which we have put within parentheses is not in Cayley's paper although it is needed as we will see in Appendix C. Plücker and Cayley used the same strategy of proof: there is a curve of degree $3(d-2)$ that intersects $\gamma$ precisely in the inflection points and similarly there is a curve of degree 
$3(4 d-9)$ that intersects $\gamma$ precisely in the sextactic points. The results then follow from Bézout's theorem.

The term sextactic point might have been introduced by Cayley in [Ca1]. Cayley remarks that sextactic points were studied before him by Plücker and Steiner without giving concrete references. He is certainly referring to papers in Crelle's Journal 32 (1846) by Steiner and 34 (1847) by Plücker. One can add a paper by Hesse in volume 36 (1848) of the same journal. In all of these papers it is claimed that there are twenty seven sextactic points on a (smooth) cubic. Steiner claims in his paper that does not contain any proofs that nine of these are always real. This is only correct as Plücker points out if the curve has two real branches. A real cubic has three sextactic points if it has only one real branch. We will discuss this in Appendix C. Plücker's paper is a polemic against Steiner and his methods in favor of analytic geometry.

A formula due to Klein implies that a smooth algebraic curve of degree $d$ in the real projective plane can have at most $d(d-2)$ inflection points, i.e., at most one third of the complex inflection points can be real. An analogous result for sextactic points seems to be unknown. A rigorous proof of Klein's formula was given by Wall in [Wa].

The content of the sections of the paper is as follows. Section 2 contains preliminaries. Section 3 explains our axiomatic approach to sextactic points. In Section 4 we give a complete proof of the results of Mukhopadhyaya since we need all the arguments involved, and a treatment of these ideas satisfying modern standards does not seem to exist. In Section 5 we prove the above theorems (Theorem 1.1 is the same as 5.2, Theorem 1.2 (ii) is in 5.3 and 5.5, (iii) is in 5.4, Theorem 1.3 is in 5.4 and 5.5.). In Appendix A we prove a theorem on simple closed curves in $P^{2}$ with few inflection points that is needed in Section 5. In Appendix B we give examples that show that the above theorems and some of the results in Section 5 are optimal. Two of these examples are due to Izumiya and Sano [IS]. In Appendix $\mathrm{C}$ we sketch a proof of the theorem of Cayley mentioned above that is based on standard results on inflection points of linear systems. What we prove is slightly more general than Cayley's result since we do not make any assumptions on the multiplicity of the inflection points of the curve. We also discuss the sextactic points on cubics in Appendix C. 


\section{$\S 2$. Preliminaries}

\section{A. Multiplicity of intersection points}

Let $\gamma$ and $\sigma$ be two smooth and regular parameterized curves in $P^{2}$. The following definitions are all of a local nature. We therefore assume that both curves are simple, i.e., without self-intersections. Assume that $p \in P^{2}$ lies in the image of both curves. Then the multiplicity of the intersection of $\gamma$ and $\sigma$ in $p$ is defined as follows. The multiplicity is equal to one if $\gamma$ and $\sigma$ intersect transversally in $p$. If they do not intersect transversally, we look at coordinates $(x, y)$ about $p$ that we assume to correspond to $(0,0)$ with the $x$-axis as the common tangent. Express the curves locally as graphs over the $x$-axis in these coordinates. Assume that the first $k$ derivatives of the $y$-components of the curves coincide in 0 , but not the $k+1$ st. Then we say that the multiplicity of the common point $p$ is equal to $k+1$ and the order of contact of $\gamma$ and $\sigma$ in $p$ is equal to $k$. If all derivatives coincide, the multiplicity and the order of contact are infinite.

We say that $\gamma$ and $\sigma$ cross in $p$ if either they meet transversally in $p$ or if $p$ is isolated in $\gamma \cap \sigma$ and there are coordinates $(x, y)$ in which $p$ corresponds to $(0,0)$, the tangent lines of both curves in $p$ corresponds to the $x$-axis, the images of $\gamma$ and $\sigma$ are locally around $p$ graphs of functions $f$ and $g$, and $f-g$ changes sign in 0 and only vanishes in 0 . We say that they are locally one on the side of the other around $p$, if they are not transversal in $p, f-g$ does not change sign in $p$ and $f-g$ does not vanish except in 0 for functions $f$ and $g$ as above.

If two curves $\gamma$ and $\sigma$ have an isolated connected set $J$ of common points, we can extend the above definition and say that the curves either cross in $J$ or are locally one on the side of the other around $J$.

If $\gamma$ and $\sigma$ meet with finite multiplicity in a point $p$, then it follows from Elementary Calculus that $p$ is isolated in the set of common points. If $\gamma$ and $\sigma$ meet with odd multiplicity in $p$, then it follows that the curves cross in $p$. If the multiplicity is even, they are locally one on the side of each other.

\section{B. Conics}

For us a conic will be a quadric without singularities. This excludes the reducible quadrics which are either a union of two lines or a line counted twice. Any two different conics are projectively equivalent.

Two conics in $P^{2}$ are identical if they have five different points in common. Given five points in $P^{2}$, no three of which are collinear, there is a 
unique conic passing through these points.

If we count common points with multiplicities in the sense defined above, it follows that two conics with five points in common coincide. The family of conics that are tangent to a curve $\gamma$ at two different points $p$ and $q$ is one dimensional, and two conics in that family only have the two given points in common. There is a one dimensional family of conics that meet a curve $\gamma$ with multiplicity at least four at a given point, and two conics in that family only have the given point in common.

\section{Inflection and sextactic points}

We will call a point $p$ on $\gamma$ an inflection point if $\operatorname{det}\left(\hat{\gamma}, \hat{\gamma}^{\prime}, \hat{\gamma}^{\prime \prime}\right)$ vanishes in $p$, where $\hat{\gamma}$ is a representation of $\gamma$ in homogeneous coordinates. An equivalent definition is to say that $p$ on $\gamma$ is an inflection point if $\gamma$ and the tangent line of $\gamma$ in $p$ meet with multiplicity at least three in $p$. We are used to think of an inflection point as a point where, roughly speaking, the direction changes in which the curve is bending. We therefore call $p$ on $\gamma$ a true inflection point, if the tangent line of $\gamma$ at $p$ and $\gamma$ cross in $p$ or if they cross in the connected component containing $p$ of their common points. It follows that the multiplicity with which the tangent line of $p$ at $\gamma$ and $\gamma$ meet is odd or infinite in $p$, if $p$ is a true inflection point.

We can now state a more precise version of the Theorem of Möbius [Mö] than in the introduction, see [TU1].

THEOREM 2.1. (Möbius) Let $\gamma$ be a simple closed curve in $P^{2}$ that is not null-homotopic and not a projective line. Then $\gamma$ has at least three intervals of true inflection points.

We will also need the following theorem. The complements of lines in $P^{2}$ are called affine planes.

THEOREM 2.2. Let $\gamma:[0,1] \rightarrow P^{2}$ be a regular simple arc such that no $\gamma(t)$ for $t \in(0,1)$ is an inflection point. Then $\gamma$ lies in an affine plane.

A contractible simple closed curve $\gamma: S^{1} \rightarrow P^{2}$ with less than four intervals of true inflection points is contained in an affine plane.

The first part of this theorem is in [Ar2], see also [Ar1]. The second part of the theorem is proved in [Ar2] for the special case of precisely two true inflection points. We give a complete proof of the second part of this proposition in Appendix A, that is based on our paper [TU1]. 
A closed curve $\gamma$ in $P^{2}$ is called convex if it lies in some affine plane in $P^{2}$ where it is convex in the usual sense of bounding a convex domain. A closed curve is called strictly convex if it is convex and has no inflection points. One can prove that a closed curve without self-intersections and inflection points in $P^{2}$ is a strictly convex closed curve. In fact, Theorem 2.2 implies that such a curve is contained in an affine plane, where the claim is standard.

Let $p$ be a point on a smooth and regular curve $\gamma$ in $P^{2}$ that is not an inflection point. Then there is a unique conic that meets $\gamma$ with multiplicity five at least in $p$, see e.g. [Bo]. This conic is called the osculating conic of $\gamma$ at $p$. It is clear that there is no regular conic meeting a curve with multiplicity five or higher in an inflection point. If the multiplicity is precisely five between a curve $\gamma$ and the osculating conic in $p$, then $\gamma$ and the osculating conic cross in $p$.

If the osculating conic of $\gamma$ at $p$ meets $\gamma$ with multiplicity six at least in $p$, then $p$ is called a sextactic point. If $\gamma$ lies in an affine plane $A^{2} \subset P^{2}$, then $p$ is sextactic if and only if $p$ is a critical point of the affine curvature of $\gamma$, see [Bl2]. The affine curvature (or the projective length element) will not play any role in the proofs of the main results of this paper and will only be referred to in some remarks.

We will need the following lemma that can already be found in [Mu1]. The books [Bl2] and [Bo] bring it as an exercise. We will give a proof of the lemma in Section 4, which applies to arcs that are only $C^{4}$.

LEMMA 2.3. Let $\gamma$ be an arc in $P^{2}$ that is free of inflection and sextactic points. Then the osculating conics at two different points of $\gamma$ do not meet.

Our methods will mostly imply the existence of sextactic points with the property that the curve and the osculating conic do not cross there. In fact, Mukhopadhyaya requires this noncrossing property in his definition of a sextactic point.

We now introduce terminology to describe the different cases of sextactic points we will encounter. Notice that a conic divides $P^{2}$ into two closed domains, one of which is a homeomorphic to a disk, the other is homeomorphic to a Möbius strip. We say that a curve is inside the conic if it lies in the disk and outside if it lies in the Möbius strip. We will call a sextactic 
point $p$ of $\gamma$ minimal if some arc of $\gamma$ around $p$ is inside of the osculating conic at $p$ and maximal if some arc around $p$ is outside the osculating conic at $p$. We will call a sextactic point of a closed curve $\gamma$ globally maximal if the whole curve $\gamma$ lies inside the osculating conic and globally minimal if it lies outside the osculating conic. A sextactic point of a closed curve $\gamma$ will be called clean if the intersection of the osculating conic and $\gamma$ is connected.

Notice that a sextactic point in which $\gamma$ and the osculating conic meet with odd multiplicity does not satisfy these additional properties we have been defining and the same can happen if the multiplicity is infinite.

The above definition of a sextactic point only makes sense for curves that are $C^{5}$ at least. A point on a $C^{4}$-regular arc is called sextactic if the osculating conic does not cross in that point. Notice that this definition implies, but is not equivalent to the original definition if the curve is $C^{5}$.

\section{$\S 3$. Intrinsic conic systems}

In this section we explain our axiomatic approach to sextactic points. It will be the main tool to prove the existence of sextactic points in the several different, although similar, situations in Sections 4 and 5. We will define an abstract notion of a sextactic point in our axiomatic setting that will turn out to correspond to those sextactic points of curves that we call maximal or minimal, see the Preliminaries.

\section{A. Intrinsic circle systems}

We will need a lemma on intrinsic circle systems. Let $I$ be either the circle $S^{1}$ or an interval of $S^{1}$ that can be open, closed or halfopen. We denote the closure of $I$ by $\bar{I}$ and the interior by $I^{\circ}$. A family $\left\{F_{p}\right\}_{p \in I}$ of closed subsets in $S^{1}$ is called an intrinsic circle system on the interval $I$ if it satisfies the following axioms:

(I0) The point $p$ is contained in $F_{p}$ for every $p \in I$.

(I1) If the set $F_{p} \cap F_{q}$ is non-empty, then $F_{p}=F_{q}$.

(I2) If $p^{\prime} \in F_{p}$ and $q^{\prime} \in F_{q}$ satisfy $p \prec q \prec p^{\prime} \prec q^{\prime}(\prec p)$, then $F_{p}=F_{q}$ holds.

(I3) Let $\left(p_{n}\right)_{n \in \mathbf{N}}$ and $\left(q_{n}\right)_{n \in \mathbf{N}}$ be two sequences in $I^{\circ}$ such that $\lim _{n \rightarrow \infty} p_{n}$ $=p$ and $\lim _{n \rightarrow \infty} q_{n}=q$ respectively where $p, q \in I$. Suppose that $q_{n} \in F_{p_{n}}$ for all $n$. Then $q \in F_{p}$ holds. 
Remark. In [Um] intrinsic circle systems were defined on the whole circle $S^{1}$. This new definition is a slight generalization to any subinterval in $S^{1}$.

We give two examples of intrinsic circle systems from the papers [Um] and [TU1].

ExAmples. (i) Let $\gamma: S^{1} \rightarrow \mathbf{R}^{2}$ be a $C^{2}$ regular simple closed curve. Let $p$ be a point on $\gamma$ and denote by $C_{p}^{\bullet}$ the largest circle in the domain bounded by $\gamma$ that touches $\gamma$ in $p$. Set

$$
F_{p}^{\bullet}=\gamma \cap C_{p}^{\bullet}
$$

It is easy to see that $\left\{F_{p}^{\bullet}\right\}_{p \in S^{1}}$ is an intrinsic circle system. One can similarly define an intrinsic circle system $\left\{F_{p}^{\circ}\right\}_{p \in S^{1}}$ using the smallest circle $C_{p}^{\circ}$ that is contained in the exterior domain of $\gamma$ and touches $\gamma$ in $p$ instead of $C_{p}^{\bullet}$, see [Um]. If $\gamma$ is $C^{3}$, then it is easy to see that the curvature of $\gamma$ has a critical point at $p$ if either $F_{p}^{\bullet}$ or $F_{p}^{\circ}$ is connected.

(ii) Let $f: P^{1} \rightarrow P^{1}$ be a diffeomorphism of the real projective line. Let $p$ be a point in $P^{1}$ and denote by $\mathcal{P}_{p}$ the one-parameter family of projective transformations of $P^{1}$ with the same 1-jet as $f$ in $p$. We assume that $\mathcal{P}_{p}$ is parameterized by the real numbers and consider $f \circ P_{t}^{-1}$ for $P_{t} \in \mathcal{P}_{p}$. Then there are two numbers $t_{0} \leq t_{1}$ such that $f \circ P_{t}^{-1}$ has only a fixed point in $p$ if $t \notin\left[t_{0}, t_{1}\right]$ and more fixed points than $p$ if $t \in\left(t_{0}, t_{1}\right)$. We assume the parameter to be chosen such that $f \circ P_{t}^{-1}$ moves points locally on the left of $p$ away and brings those locally on the right of $p$ closer if $t<t_{0}$. Let $F_{p}^{\bullet}$ denote the fixed point set of $f \circ P_{t_{0}}^{-1}$ and $F_{p}^{\circ}$ the fixed point set of $f \circ P_{t_{1}}^{-1}$. It is proved in [TU1] that $\left\{F_{p}^{\bullet}\right\}_{p \in P^{1}}$ and $\left\{F_{p}^{\circ}\right\}_{p \in P^{1}}$ are intrinsic circle systems. A point $p$ is called a projective point of $f$ if there is a projective transformation that has the same 3 -jet as $f$ in $p$. In general there is only a projective transformation with the same 2-jet as $f$ at a point $p$. If either $F_{p}^{\bullet}$ or $F_{p}^{\circ}$ is connected, then $p$ is a projective point.

The following basic but easy lemma is proved in [Um] for an intrinsic circle systems on $S^{1}$. The proof in the more general case is exactly the same. Notice that the idea behind the lemma is essentially due to H. Kneser [Kn], although not in this abstract setting. One does not need Axiom (I3) in the proof of the lemma. 
LEMMA 3.1. Let $\left\{F_{p}\right\}_{p \in I}$ be an intrinsic circle system on $I=[a, b]$. Suppose that $F_{a}=F_{b}$ and $F_{a} \cap(a, b)$ is empty. Then there exists a point $c \in(a, b)$ such that $F_{c}$ is connected and contained in $(a, b)$.

Lemma 3.1 applied to Example (i) has the classical Four-Vertex Theorem as a consequence and is nothing but a reformulation of its proof in [Kn]. Applied to Example (ii), the theorem of Ghys that a diffeomorphism of $P^{1}$ has at least four projective points follows, see [TU1].

\section{B. Intrinsic conic systems}

We will define an intrinsic conic system on an interval $I$ of $S^{1}$ to be a set of functions from $S^{1} \times S^{1}$ into the nonnegative even integers extended by $\infty$ that are indexed by a subset of $\bar{I} \times \bar{I}$ and satisfy certain axioms. On one hand this is analogous to the intrinsic circle systems defined above. On the other hand it is related to divisors on complex algebraic curves. As we will see in Appendix $\mathrm{C}$, given a plane algebraic curve, one can consider the linear system of divisors that come from intersections of the curve with conics and use it to prove the formula of Cayley for the number of sextactic points mentioned in the introduction. The intrinsic conic systems that we consider here do not correspond to the whole linear system, but only to those coming from intersections with conics that are tangent to the curve and do not cross it at any of the common points. This noncrossing property is the reason why we restrict ourselves to even or infinite values of the functions. See Example (i) that we give after the axioms and the next section for full details of this application. Generalizations to higher order intrinsic systems and applications to Fourier series of periodic functions will be given in [TU2]. We explain a special case of the construction in [TU2] in Example (ii) after the axioms.

Let $I$ be either the circle $S^{1}$ or an interval of $S^{1}$ that can be open, closed or halfopen. To avoid trivialities, we assume that the length of $I$ is positive. We set

$$
I_{*}^{2}:=(\bar{I} \times \bar{I}) \backslash\{(p, p) \mid p \in \bar{I} \backslash I\}
$$

i.e., $I_{*}^{2}$ is the closed square $\bar{I} \times \bar{I}$ with a corner point $(p, p)$ removed if $p \notin I$. A family $\left\{f_{p, q}\right\}_{(p, q) \in I_{*}^{2}}$ of functions $f_{p, q}: S^{1} \rightarrow 2 \mathbf{N}_{0} \cup\{\infty\}$, where $2 \mathbf{N}_{0}$ denotes the nonnegative even integers, is called an intrinsic conic system on the interval $I$ if it satisfies the axioms that will be listed below. (It is important that the functions $f_{p, q}$ be defined on the whole circle $S^{1}$ since 
Axiom (A7) below might otherwise be violated in the applications.) Notice that $f_{p, q}$ is defined for $p \neq q$ if and only if $p, q \in \bar{I}$ and $f_{p, p}$ is defined if and only if $p \in I$. We will denote the support of $f_{p, q}$ by $F_{p, q}$, i.e.,

$$
F_{p, q}=\left\{r \in S^{1} \mid f_{p, q}(r)>0\right\}
$$

The value of $f_{p, q}$ at a point $r$ will be called the multiplicity of $r$ with respect to $f_{p, q}$. The sum over all values of $f_{p, q}$, which can of course be infinite, is called the total multiplicity of $f_{p, q}$. A point $r$ in $S^{1}$ will be called sextactic if its multiplicity with respect to some $f_{p, q}$ is at least six. We now list the axioms and follow them by examples that explain their geometric meaning.

(A1) $F_{p, q}$ is closed and $p, q \in F_{p, q}$ for all $(p, q) \in I_{*}^{2}$.

(A2) $f_{p, q}=f_{q, p}$ for all $(p, q) \in I_{*}^{2}$.

(A3) If $F_{p, q}$ and $F_{p, r}$ have a point $s \neq p$ in common, then $f_{p, q}=f_{p, r}$.

(A4) If $p^{\prime \prime} \in F_{p, p^{\prime}}$ and $q^{\prime \prime} \in F_{q, q^{\prime}}$ satisfy $p \preceq q \preceq p^{\prime} \preceq q^{\prime} \prec p^{\prime \prime} \prec q^{\prime \prime}(\prec p)$ or $p \succeq q \succeq p^{\prime} \succeq q^{\prime} \succ p^{\prime \prime} \succ q^{\prime \prime}(\succ p)$, and $f_{p, p^{\prime}}(p) \geq 4$ if $p=p^{\prime}$, and $f_{q, q^{\prime}}(q) \geq 4$ if $q=q^{\prime}$, then $f_{p, p^{\prime}}=f_{q, q^{\prime}}$ holds.

(A5) If $f_{p, q}(r) \geq 4$ and $r \in I$, then $f_{r, r}=f_{p, q}$.

(A6) Let $\left(\left(p_{n}, q_{n}\right)\right)$ be a sequence in $I_{*}^{2}$ such that $\lim _{n \rightarrow \infty}\left(p_{n}, q_{n}\right)=(p, q) \in$ $I_{*}^{2}$, and let $\left(r_{n}^{1}\right)$ and $\left(r_{n}^{2}\right)$ be two sequences such that $r_{n}^{i} \in \bar{I} \cap F_{p_{n}, q_{n}}$ and $\lim _{n \rightarrow \infty} r_{n}^{i}=r \in \bar{I}$ for $i=1,2$. Assume $f_{p_{n}, q_{n}}\left(r_{n}^{1}\right) \geq k_{1}$ and $f_{p_{n}, q_{n}}\left(r_{n}^{2}\right) \geq k_{2}$ for all $n$. Then $f_{p, q}(r) \geq \max \left\{k_{1}, k_{2}\right\}$, and the inequality is strict if $r_{n}^{1}$ and $r_{n}^{2}$ are different for all $n$.

(A7) The total multiplicity of $f_{p, q}$ is at least six for all $(p, q) \in I_{*}^{2}$.

(A8) If $f_{p, q}(p)=2$, then $p$ is isolated in $F_{p, q}$.

ExAmPles. (i) Let $\gamma$ be a strictly convex curve in the affine plan. We identify $\gamma$ with $S^{1}$. Let $C$ be a conic. Then we can associate to $C$ a function on $S^{1}$ that associates to a point $r$ on $\gamma$ the multiplicity with which $C$ and $\gamma$ meet in $r$. The multiplicity is of course zero in points in which $C$ and $\gamma$ do not meet. Let $I$ denote $S^{1}$ or some interval on $S^{1}$ and let $(p, q) \in I_{*}^{2}$. If $p \neq q$, we let $C_{p, q}$ denote the maximal inscribed conic that is tangent to $\gamma$ in $p$ and $q$. If $p=q$, we let $C_{p, q}$ denote the maximal inscribed conic that meets $\gamma$ with multiplicity at least four in $p$. We let $f_{p, q}$ denote the function corresponding to $C_{p, q}$ as explained above. We will prove in Section 3 that $\left\{f_{p, q}\right\}_{(p, q) \in I_{*}^{2}}$ is an intrinsic conic system. The sextactic points of $\left\{f_{p, q}\right\}_{(p, q) \in I_{*}^{2}}$ are precisely the globally maximal sextactic points of $\gamma$. 
(ii) For a real valued $C^{4}$-function $u$ on $S^{1}$, one defines the osculating polynomial $\varphi_{s}$ (of order five) at a point $s \in S^{1}$ to be the unique trigonometric polynomial of degree two,

$$
\varphi_{s}(t)=a_{0}+a_{1} \cos t+b_{1} \sin t+a_{2} \cos 2 t+b_{2} \sin 2 t,
$$

whose value and first four derivatives at $s$ coincide with those of $u$ at $s$. If $u$ is $C^{5}$ and the value and the first five derivatives of $u$ and $\varphi_{s}$ coincide in $s$, i.e., if $\varphi_{s}$ hyperosculates $u$ in $s$, then we call $s$ a flex of $u$ (of order five). The existence of six flexes of order five can easily be proved as a consequence of the well-known fact that a function has at least six zeros if its Fourier coefficients $a_{i}$ and $b_{i}$ vanish for $i \leq 2$, see [TU2]. One can use intrinsic conic systems to prove the much stronger result that there are six such flexes satisfying the global property that the osculating polynomials $\varphi_{s}$ in the flexes support $u$, i.e., either $\varphi_{s} \leq u$ or $u \leq \varphi_{s}$. The intrinsic conic systems are defined as follows. To simplify the definition we assume $u$ to be $C^{\infty}$. Let $(p, q) \in S^{1} \times S^{1}$. If $p \neq q$, we let $\varphi_{p, q}$ denote the smallest trigonometric polynomial of degree two that is greater or equal to $u$ and has the same values as $u$ in $p$ and $q$. If $p=q$ we let $\varphi_{p, q}$ denote the smallest trigonometric polynomial of degree two that is greater or equal to $u$ and has the same 1-jet as $u$ in $p=q$. We now define $f_{p, q}: S^{1} \rightarrow 2 \mathbf{N}_{0} \cup\{\infty\}$ by setting $f_{p, q}(r)=2 k$ if the $2 k-1$-jets of $u$ and $\varphi_{p, q}$ agree in $r$ but not the $2 k$-jets, $f_{p, q}(r)=0$ if the values of $u$ and $\varphi_{p, q}$ do not agree in $r$, and $f_{p, q}(r)=\infty$ otherwise. One can now prove that $\left\{f_{p, q}\right\}_{(p, q) \in S^{1} \times S^{1}}$ is an intrinsic conic system and similarly define an intrinsic conic system using trigonometric polynomials that are smaller or equal to $u$. We refer to [TU2] for much more general results concerning Fourier polynomials of arbitrary degree. Notice that Fourier polynomials of degree one lead to intrinsic circle system.

We now start deriving consequences of the axioms of an intrinsic conic system. The following lemma is trivial.

LEMMA 3.2. Let $\left\{f_{p, q}\right\}_{(p, q) \in I_{*}^{2}}$ be an intrinsic conic system on an interval $I$ in $S^{1}$. Then for any subinterval $J$ of $I$, the restriction $\left\{f_{p, q}\right\}_{(p, q) \in J_{*}^{2}}$ is an intrinsic conic system on $J$.

The following lemma is an immediate consequence of Axiom (A7).

LEMmA 3.3. If $F_{p, p}$ only consists of the point $p$, then $p$ is sextactic. 
We also have the following two easy lemmas.

LEMMA 3.4. $f_{p, p}(p) \geq 4$ for every $p \in I$.

Proof. Let $\left(p_{n}\right)$ and $\left(q_{n}\right)$ be two sequences in $I$ that converge to $p$ and $p_{n} \neq q_{n}$ for all $n$. Applying (A6) to the situation $r_{n}^{1}=p_{n}$ and $r_{n}^{2}=q_{n}$, we get $f_{p, p}(p)>2$ since $p_{n} \in F_{p_{n}, q_{n}}$ and $q_{n} \in F_{p_{n}, q_{n}}$ by (A1). Using that $f_{p, p}(p)$ is an even number, we get $f_{p, p}(p) \geq 4$.

LEMMA 3.5. If $\left(p_{n}\right)$ and $\left(q_{n}\right)$ are sequences in $I^{\circ}$ that both converge to $p \in I, q_{n} \in F_{p_{n}, p_{n}}$, and $q_{n}$ is different from $p_{n}$, then $p$ is sextactic.

Proof. By Lemma 3.4, we have $f_{p_{n}, p_{n}}\left(p_{n}\right) \geq 4$ for every $n$. Hence (A6) implies that $f_{p, p}(p)>4$ since we can choose $r_{n}^{1}=p_{n}$ and $r_{n}^{2}=q_{n}$. It follows that $f_{p, p}(p) \geq 6$ and hence that $p$ is sextactic.

Lemma 3.6. If $r \in F_{p, q} \cap I$ is not isolated in $F_{p, q} \cap I$, then $r$ is a sextactic point with infinite multiplicity with respect to $f_{p, q}$.

Proof. We assume that $f_{p, q}(r)$ is a finite number $k$. Let $\left(r_{n}\right)$ be a sequence in $F_{p, q}$ of pairwise different points that are all different from $r$ and converges to $r$. We now apply (A6) to the situation $p_{n}=p, q_{n}=q$, $r_{n}^{1}=r$ and $r_{n}^{2}=r_{n}$. It follows that $f_{p, q}(r)>k$, a contradiction. Hence $f_{p, q}(r)=\infty$.

If the support of $f_{p, p}$ is a connected set, then we say that $p$ is a clean sextactic point. Lemmas 3.3 and 3.6 imply that a clean sextactic point in $I$ is sextactic and that moreover every point in the intersection of the support of $f_{p, p}$ with $I$ is a clean sextactic point if $p$ is.

LEMMA 3.7. If $F_{p, q} \neq F_{q, q}$, then $f_{p, q}(q)=2, q$ is isolated in $F_{p, q}$ and $F_{p, q}$ has at least two components.

Proof. If $F_{p, q} \neq F_{q, q}$, then (A5) implies that $f_{p, q}(q)=2$. By (A8), we know that $q$ is isolated in $F_{p, q}$. Since $q$ must of course be different from $p$, we see that $F_{p, q}$ must have at least two connected components.

We set

$$
F_{p, q}^{*}= \begin{cases}F_{p, q} & \text { if } f_{p, q}(p) \geq 4 \\ F_{p, q} \backslash\{p\} & \text { if } f_{p, q}(p)=2\end{cases}
$$


LEMMA 3.8. Let $I$ be $S^{1}$ or an interval on $S^{1}$ and $\left\{f_{p, q}\right\}_{(p, q) \in I_{*}^{2}}$ an intrinsic conic system on $I$. Then for each $p \in I,\left\{F_{p, q}^{*}\right\}_{q \in \bar{I}}$ is an intrinsic circle system on $\bar{I}$.

Proof. First notice that $f_{p, q}$ is defined for all $q \in \bar{I}$ since $p \in I$. To prove (I0), notice that $q \in F_{p, q}^{*}$ if $q \neq p$. If $q=p$, then $f_{p, q}(p)=f_{p, p}(p) \geq 4$ and hence $q \in F_{p, q}=F_{p, q}^{*}$.

To prove (I1) assume that $s \in F_{p, q}^{*}=F_{p, r}^{*}$. If $s=p$, then $f_{p, q}(p) \geq 4$ and $f_{p, r}(p) \geq 4$ and hence $f_{p, q}=f_{p, s}$ by (A5). Now assume that $s \neq p$. Then (A3) implies that $f_{p, q}=f_{p, s}$.

Property (I2) follows from (A4).

To prove that (I3) holds, let $\left(q_{n}\right)$ and $\left(r_{n}\right)$ be sequences in $I^{\circ}$ with limits $q$ and $r \in \bar{I}$ respectively, and assume that $r_{n} \in F_{p, q_{n}}^{*}$, then it follows from (A6) that $r \in F_{p, q}$ and (I3) follows if $r \neq p$. If $r=p$, then we have to prove that $f_{p, q}(p) \geq 4$. If only finitely many of the $r_{n}$ are equal to $p$, then (A6) applied to $r_{n}$ and the constant sequence $p$ implies that $f_{p, q}(p) \geq 4$. If infinitely many of the $r_{n}$ are equal to $p$, then for these $r_{n}$ we have $f_{p, q_{n}}(p) \geq 4$ and hence that $f_{p, q}(p) \geq 4$.

LEMmA 3.9. Let $I=[a, b]$ or $[a, b), a \prec b$, be an interval on $S^{1}$, $\left\{f_{p, q}\right\}_{(p, q) \in I_{*}^{2}}$ an intrinsic conic system on $I$ such that $f_{a, a}=f_{a, b}$ and $F_{a, b} \cap(a, b)$ is empty. Then there exists a point $c \in(a, b)$ such that $f_{a, c}=f_{c, c}$ and $F_{c, c}$ is contained in $[a, b)$. Furthermore, $a$ is isolated in $F_{a, c}$ and $F_{a, c}$ has exactly two components.

Similarly, if $I=[a, b]$ or $(a, b], f_{b, b}=f_{a, b}$ and $F_{a, b} \cap(a, b)$ is empty, then there exists a point $c \in(a, b)$ such that $f_{b, c}=f_{c, c}$ and $F_{c, c}$ is contained in $(a, b]$. Furthermore, $b$ is isolated in $F_{b, c}$ and $F_{b, c}$ has exactly two components.

Proof. We only prove the first part; the second part is similar. By Lemma 3.8 we know that $\left\{F_{a, q}^{*}\right\}_{q \in \bar{I}}$ is an intrinsic circle system on $\bar{I}$. Clearly it satisfies $F_{a, a}^{*}=F_{a, b}^{*}$. Hence there exists a point $c \in(a, b)$ by Lemma 3.1 such that $F_{a, c}^{*}$ is connected and contained in $(a, b)$. Hence $a \notin F_{a, c}^{*}$ which implies that $F_{a, a} \neq F_{a, c}$ and $F_{a, c}$ has exactly two connected components. Therefore we have $F_{a, c}=F_{c, c}$ and hence $f_{a, c}=f_{c, c}$. It is clear that $F_{c, c}$ is contained in $[a, b)$.

LEMmA 3.10. Let $I=[a, b]$ or $[a, b), a \prec b$, be a closed interval of $S^{1}$ and $\left\{f_{p, q}\right\}_{(p, q) \in I_{*}^{2}}$ be an intrinsic conic system on $I$. Suppose that $f_{a, a}=f_{a, b}$ and $F_{a, b} \cap(a, b)$ is empty. Then there exist two distinct points $a_{1} \prec b_{1}$ in 
$(a, b)$ such that $f_{a_{1}, b_{1}}=f_{a_{1}, a_{1}}$ and $F_{a_{1}, b_{1}}=F_{a_{1}, a_{1}} \subset(a, b), b_{1}$ is isolated in $F_{a_{1}, b_{1}}$ and $F_{a_{1}, b_{1}}$ has exactly two components.

Similarly, if $I=[a, b]$ or $(a, b], f_{b, b}=f_{a, b}$ and $F_{a, b} \cap(a, b)$ is empty, then there exist two distinct points $a_{1} \prec b_{1}$ in $(a, b)$ such that $f_{a_{1}, b_{1}}=f_{b_{1}, b_{1}}$ and $F_{a_{1}, b_{1}}=F_{b_{1}, b_{1}} \subset(a, b), a_{1}$ is isolated in $F_{a_{1}, b_{1}}$ and $F_{a_{1}, b_{1}}$ has exactly two components.

Proof. We prove the first part of the lemma. By Lemma 3.9, there exists a point $b_{1} \in(a, b)$ such that $f_{a, b_{1}}=f_{b_{1}, b_{1}}$ with support in $I$ and $a$ is isolated in $F_{a, b_{1}}$. We can assume that $b_{1}$ is such that $\left(a, b_{1}\right) \cap F_{a, b_{1}}=\emptyset$. Then by Lemma 3.9 , there exists a point $a_{1} \in\left(a, b_{1}\right)$ such that $f_{a_{1}, b_{1}}=f_{a_{1}, a_{1}}$ with support in $\left(a, b_{1}\right] \subset(a, b)$.

The next two propositions will be the main tools to find sextactic points in Sections 4 and 5.

Proposition 3.11. Let $I$ be a closed or halfopen interval of $S^{1}$ with endpoints $a$ and $b$ and let $\left\{f_{p, q}\right\}_{(p, q) \in I_{*}^{2}}$ be an intrinsic conic system on $I$. Suppose that $F_{a, b} \cap(a, b)$ is empty. We assume furthermore that either $f_{a, b}=f_{a, a}$ or $f_{a, b}=f_{b, b}$ holds (at least one of these conditions makes sense when $I$ is halfopen). Then there is a sextactic point $r$ in $(a, b)$.

Proof. Let $J=\left[a_{1}, b_{1}\right]$ be an interval as in Lemma 3.10. Let $\mathcal{C}_{J}$ denote the set of $(\alpha, \beta) \in(a, b) \times(a, b)$ such that $\alpha \neq \beta, f_{\alpha, \beta}=f_{\alpha, \alpha}$ with support in $J, F_{\alpha, \beta} \cap I(\alpha, \beta)=\emptyset$, and $F_{\alpha, \beta}$ consists of precisely two components, one of which is the isolated point $\beta$. Here $I(\alpha, \beta)$ denotes the open interval with endpoints $\alpha$ and $\beta$. (Notice that we do not assume that $\alpha<\beta$.) We know from Lemma 3.10 that $\mathcal{C}_{J}$ is nonempty.

We let $\delta_{\alpha, \beta}$ denote the distance between $\alpha$ and $\beta$. Let $\delta$ denote the infimum over $\delta_{\alpha, \beta}$ for $(\alpha, \beta) \in \mathcal{C}_{J}$.

We consider a sequence $\left\{\left(\alpha_{n}, \beta_{n}\right)\right\}$ in $\mathcal{C}_{J}$ such that $\delta_{\alpha_{n}, \beta_{n}}$ converges to $\delta$. By going to subsequences if necessary, we may assume that

$$
\lim _{n \rightarrow \infty} \alpha_{n}=\alpha, \quad \lim _{n \rightarrow \infty} \beta_{n}=\beta \text {. }
$$

If $\alpha=\beta$, then it follows immediately from Lemma 3.5 that $\alpha$ is a sextactic point. We can therefore assume that $\delta>0$. To simplify the notation we will assume that $\alpha \prec \beta$. By (A6), we have $f_{\alpha, \alpha}(\alpha) \geq 4$ and $f_{\alpha, \beta}(\alpha) \geq 4$ and hence $f_{\alpha, \alpha}=f_{\alpha, \beta}$ by (A5). (We do not claim that $F_{\alpha, \beta}$ is contained in $J$.) We can assume that $\alpha$ and $\beta$ are isolated in $F_{\alpha, \beta}$ since otherwise we have a 
sextactic point by Lemma 3.6. Let $\beta^{\prime}$ be the point in $F_{\alpha, \beta} \cap(\alpha, \beta]$ closest to $\alpha$. We now apply Lemma 3.9 to the interval $\left[\alpha, \beta^{\prime}\right]$ and we find a point $\gamma \in\left(\alpha, \beta^{\prime}\right)$ such that $(\gamma, \alpha) \in \mathcal{C}_{J}$. Clearly $\delta_{\gamma, \alpha}<\delta$, which is a contradiction. Hence there is a sextactic point in $J \subset(a, b)$.

Proposition 3.12. Let $\left\{f_{p, q}\right\}_{(p, q) \in I_{*}^{2}}$ be an intrinsic conic system on $I$, where $I$ is some interval on $S^{1}$ or the whole circle $S^{1}$. Assume that $p$ and $q$ are contained in distinct components of $F_{p, q}$ and that there is a third component of $F_{p, q}$ between $p$ and $q$ on $I$. Then there is a point $r \in I$ such that $r \neq q$ and $F_{r, q}$ has two connected components one of which is $\{q\}$.

Proof. We can assume without loss of generality that there is a point $p^{\prime}$ in $F_{p, q} \cap I$ different from both $p$ and $q$ such that the open interval $J$ between $p$ and $p^{\prime}$ on $I$ does not meet $F_{p, q}$. By Lemma 3.8, $\left\{F_{q, x}^{*}\right\}_{x \in I}$ is an intrinsic circle system on $I$. Since $p, p^{\prime} \in F_{q, p}^{*}$ there is by Lemma 3.1 a point $r$ in $J$ such that $F_{q, r}^{*}$ is connected and contained in $J$. Then $F_{q, r}$ has two connected components one of which is $\{q\}$.

The last two propositions were the main technical results of this section. We use them to prove the following theorem.

THEOREM 3.13. Let $\left\{f_{p, q}\right\}_{(p, q) \in S^{1} \times S^{1}}$ be an intrinsic conic system on $S^{1}$. Then $\left\{f_{p, q}\right\}_{(p, q) \in S^{1} \times S^{1}}$ has at least three sextactic points.

Remark. This theorem is optimal as the intrinsic conic system $\left\{f_{p, q}^{\bullet}\right\}_{(p, q) \in S^{1} \times S^{1}}$ of a contractible branch of a real regular cubic shows, see Section 4 and Appendix C.

Proof. We first prove the existence of one sextactic point. Let $p$ be a point on $\gamma$ that we can assume not to be sextactic. Then $f_{p, p}(p)=4$. Hence $p$ is isolated in $F_{p, p}$. We therefore have a point $q$ in $F_{p, p}$ that is different from $p$ and such that the open interval $(q, p)$ does not meet $F_{p, p}$. Proposition 3.11 now implies that there is a sextactic point $s$ in the interval $(q, p)$.

To prove that there are two further sextactic points we proceed as follows. Let $r$ be some point different from $s$. If $F_{r, s}$ consists of two components we have two sextactic points different from $s$ by Proposition 3.11. If $F_{r, s}$ consists of three components at least, we can use Proposition 3.12 to find a point $r^{\prime}$ such that $F_{r^{\prime}, s}$ consists of two components, and the existence of the two new sextactic points follows again from Proposition 3.11. 


\section{§4. An application to strictly convex curves}

In this section we use the theory of intrinsic conic systems to give a complete proof of the results of Mukhopadhyaya in [Mu2] on the existence of inscribed and circumscribed osculating conics of strictly convex curves in an affine plane. As was pointed out in Section 2, such a curve is the same thing as a simple closed curve in $P^{2}$ without inflection points.

THEOREM 4.1. (Mukhopadhyaya) Let $\gamma$ be a strictly convex curve in the affine plane $A^{2}$. Then $\gamma$ has at least three circumscribed osculating conics and at least three inscribed osculating ellipses. In particular, $\gamma$ has at least six sextactic points that are globally maximal or minimal.

The osculating conic at a point $p$ of a curve $\gamma$ is an ellipse if and only if the affine curvature of $\gamma$ at $p$ is positive. It therefore follows from the theorem that a strictly convex curve must have points with positive affine curvature.

We first point out that the theorem has an interesting corollary which does not seem to follow from the other proofs of the existence of sextactic points. We will denote the open disk which a Jordan curve $\gamma$ in $A^{2}$ bounds by $D_{\gamma}$ and refer to it as the interior domain of $\gamma$. We let $\kappa_{M}$ denote the maximum of the affine curvature of $\gamma, \kappa_{m}$ its minimum and $A\left(D_{\gamma}\right)$ the area of $D_{\gamma}$.

Corollary 4.2. Let $\gamma$ be a strictly convex curve in the affine plane $A^{2}$. Then $\kappa_{M}>0$ and

$$
\pi \kappa_{M}^{-3 / 2} \leq A\left(D_{\gamma}\right)
$$

with an equality if and only if $\gamma$ is an ellipse. If the affine curvature of $\gamma$ is positive then we also have

$$
A\left(D_{\gamma}\right) \leq \pi \kappa_{m}^{-3 / 2}
$$

with an equality if an and only if $\gamma$ is an ellipse.

Remark. Both inequalities follow from Exercises 4 and 15 in Section $\S 27$ of [Bl2] if the affine curvature is positive.

Proof of the Corollary. We already observed that $\kappa_{M}>0$. Now let $C$ be one of the inscribed osculating ellipses and denote its affine curvature by $\kappa$. Then $\kappa \leq \kappa_{M}$. The area of the interior domain of $C$ is $\pi \kappa^{-3 / 2}$. 
The first inequality follows immediately. The second inequality follows by arguing similarly with one of the circumscribed conics which must be an ellipse since the affine curvature is positive.

Before proving Theorem 4.1 we need to introduce the relevant intrinsic conic systems.

We say that the ellipse $C_{1}$ is contained in the ellipse $C_{2}$ if $D_{C_{1}} \subset D_{C_{2}}$ where $D_{C_{i}}$ is the interior domain of $C_{i}$. An inscribed ellipse is said to be maximal if it is not strictly contained in any other inscribed ellipse.

Let $p, q$ be two different points on $\gamma$. Let $\Gamma_{p, q}$ be the one dimensional family of ellipses that is tangential to $\gamma$ in $p$ and $q$. In one direction, this family converges to the closed line segment $\overline{p q}$. Since $\overline{p q}$ meets $\gamma$ transversally in $p$ and $q$, we have inscribed ellipses in the family. Thus there is a unique maximal inscribed ellipse in the family $\Gamma_{p, q}$ that we will denote by $C_{p, q}^{\bullet}$

We can also define the maximal inscribed ellipse $C_{p, q}^{\bullet}$ when $p=q$. Fix a point $p$ on $\gamma$. Let $\Gamma_{p, p}$ be the one dimensional family of ellipses that is tangential to $\gamma$ in $p$ with multiplicity at least four. The osculating ellipse of $\gamma$ in $p$ is defined since $p$ is not an inflection point and it is of course contained in the family $\Gamma_{p, p}$. No ellipse in $\Gamma_{p, p}$ can cross $\gamma$ in $p$ except possibly the osculating ellipse. In one direction, this family $\Gamma_{p, p}$ converges to the point $p$. In that same direction after passing the osculating ellipse, the ellipses lie locally around $p$ inside of $\gamma$. Hence we have inscribed ellipses in the family. There is therefore a unique maximal inscribed ellipse in the family $\Gamma_{p, p}$ that we denote by $C_{p, p}^{\bullet}$.

For any pair of points $(p, q) \in S^{1} \times S^{1}=\gamma \times \gamma$, let $f_{p, q}^{\bullet}: S^{1} \rightarrow 2 \mathbf{N}_{0} \cup\{\infty\}$ denote the function that associates to a point $r \in S^{1}=\gamma$ the multiplicity with which $C_{p, q}^{\bullet}$ and $\gamma$ meet in $r$. If $r \notin C_{p, q}^{\bullet}$, then of course $f_{p, q}^{\bullet}(r)=0$.

The following is obvious:

The functions $f_{p, q}^{\bullet}$ satisfy Axioms (A1), (A2), (A3), (A5) and (A8) for intrinsic conic systems for all $(p, q) \in S^{1} \times S^{1}$.

Notice that Axiom (A4) is an easy consequence of the fact that two ellipses cannot meet in more than four points without being identical. Hence we have: 
The functions $f_{p, q}^{\bullet}$ satisfy Axiom (A4) for intrinsic conic systems for every pair $(p, q) \in S^{1} \times S^{1}$.

Assume that $C$ is an ellipse that meets $\gamma$ in a point $p$ with multiplicity two. Then $C$ and $\gamma$ do not cross in $p$ and there is another ellipse $C^{\prime}$ tangent to $\gamma$ in $p$ and containing $C$ which locally around $p$ lies between $\gamma$ and $C$. This implies the following lemma.

Lemma 4.3. Let $p$ and $q$ be two distinct points on $\gamma$. Then the maximal ellipse $C_{p, q}^{\bullet}$ can be characterized as the only inscribed ellipse that meets $\gamma$ in $p$ and $q$ and satisfies one of the following two properties.

(i) $C_{p, q}^{\bullet}$ meets $\gamma$ in at least three different points.

(ii) $C_{p, q}^{\bullet}$ meets $\gamma$ with multiplicity at least four either in $p$ or in $q$ and it does not have any other points in common with $\gamma$.

In particular, $C_{p, q}^{\bullet}$ meets $\gamma$ with total multiplicity six at least.

The following lemma follows from the fact that if $C$ is an ellipse in $\Gamma_{p, p}$ that lies locally around $p$ inside of $\gamma$ and meets $\gamma$ in $p$ precisely with multiplicity four, then there is a different ellipse $C^{\prime}$ in $\Gamma_{p, p}$ that contains $C$, is contained in the osculating ellipse at $p$, lies locally around $p$ inside of $\gamma$ and also meets $\gamma$ in $p$ with precisely multiplicity four.

LEMmA 4.4. Let $p$ be an arbitrary point on $\gamma$. Then the maximal ellipse $C_{p, p}^{\bullet}$ can be characterized as the only inscribed ellipse that meets $\gamma$ in $p$ with multiplicity at least four and satisfies one of the following two properties.

(i) $C_{p, p}^{\bullet}$ meets $\gamma$ in at least two different points.

(ii) $C_{p, p}^{\bullet}$ meets $\gamma$ in $p$ with multiplicity at least six and has no other points in common with $\gamma$. It follows that $C_{p, p}^{\bullet}$ is the osculating ellipse at $p$ and $p$ is a sextactic point.

In particular, $C_{p, p}^{\bullet}$ meets $\gamma$ with total multiplicity six at least.

We have the following immediate corollary of Lemmas 4.3 and 4.4 .

The functions $f_{p, q}^{\bullet}$ satisfy Axiom (A7) for intrinsic conic systems for every pair $(p, q) \in S^{1} \times S^{1}$.

It is therefore only left to prove that Axiom (A6) is satisfied. 
LEMma 4.5. The functions $f_{p, q}^{\bullet}$ satisfy Axiom (A6) for intrinsic conic systems for every pair $(p, q) \in S^{1} \times S^{1}$.

Proof. We consider a small interval around $r=\gamma\left(t_{0}\right)$ on the curve $\gamma$ and parallel coordinates $(x, y)$ in which $\gamma$ on this interval corresponds to points on the $x$-axis. Let $r_{n}^{1}=\gamma\left(t_{n}^{1}\right)$ and $r_{n}^{2}=\gamma\left(t_{n}^{2}\right)$ be two sequences converging to $r$ and assume that $r_{n}^{1}$ and $r_{n}^{1} \in C_{p_{n}, q_{n}}^{\bullet}$ where $p_{n}$ and $q_{n}$ converge to $p$ and $q$ respectively. We assume that $f_{p_{n}, q_{n}}^{\bullet}\left(r_{n}^{i}\right) \geq k_{i}$ for $i=$ 1,2. Assume that $k_{1} \leq k_{2}$. We can write $C_{p_{n}, q_{n}}^{\bullet}$ locally around $r$ as a graph of a function $g_{n}(t)$ in the parallel coordinates for sufficiently big $n$. We can assume after going to a subsequence if necessary that the $C_{p_{n}, q_{n}}^{\bullet}$ converge to an ellipse $C$ that is the graph of a function $g$ in the parallel coordinates. We have that $g_{n}\left(t_{n}^{1}\right)=g_{n}^{\prime}\left(t_{n}^{1}\right)=\cdots=g_{n}^{\left(k_{1}-1\right)}\left(t_{n}^{1}\right)=0$ and $g_{n}\left(t_{n}^{2}\right)=g_{n}^{\prime}\left(t_{n}^{2}\right)=\cdots=g_{n}^{\left(k_{2}-1\right)}\left(t_{n}^{2}\right)=0$. By taking limits it clearly follows that $g\left(t_{0}\right)=g^{\prime}\left(t_{0}\right)=\cdots=g^{\left(k_{2}-1\right)}\left(t_{0}\right)=0$. Hence $C$ and $\gamma$ meet in $r$ with multiplicity $k_{2}$ at least.

We now prove that $C$ and $\gamma$ meet in $r$ with multiplicity greater than $k_{2}$ when $r_{n}^{1}$ and $r_{n}^{2}$ are different for all $n$. Set $i=k_{2}-k_{1}$. There is by Rolle's Theorem of Elementary Calculus for every $n$ an $s_{n}^{1}$ between $t_{n}^{1}$ and $t_{n}^{2}$ such that $g_{n}^{\left(k_{1}\right)}\left(s_{n}^{1}\right)=0$. Similarly we find an $s_{2}^{2}$ between $s_{n}^{1}$ and $t_{n}^{2}$ with $g_{n}^{\left(k_{1}+1\right)}\left(s_{n}^{2}\right)=0$, and inductively an $s_{n}^{j}$ between $s_{n}^{j-1}$ and $t_{n}^{2}$ with $g_{n}^{\left(k_{1}+j-1\right)}\left(s_{n}^{j}\right)=0$ for $j=1, \ldots, i+1$. Taking limits we obtain $g\left(t_{0}\right)=$ $g^{\prime}\left(t_{0}\right)=\cdots=g^{\left(k_{2}\right)}\left(t_{0}\right)=0$. This proves that $C$ and $\gamma$ meet in $r$ with multiplicity greater than $k_{2}$.

The claim in the lemma now follows after we prove that $C_{p, q}^{\bullet}$ meets $\gamma$ in $r$ at least with the same multiplicity as $C$. Notice that $C$ contains the points $p$ and $q$ and is inscribed in $\gamma$. If $p \neq q$, then it follows that $C_{p, q}^{\bullet}$ lies between $\gamma$ and $C$ since it is maximal with this property. Hence $C_{p, q}^{\bullet}$ meets $\gamma$ at least with the same multiplicity in $r$ as $C$ if $p \neq q$. If $p=q$, the same follows if we can show that $C$ meets $\gamma$ with multiplicity at least four in $p=q$. If there are infinitely many $n$ such that $p_{n}=q_{n}$, then this follows as in the first paragraph of the proof. If there are infinitely many $n$ such that $p_{n} \neq q_{n}$, this follows as in the second paragraph of the proof.

With help of circumscribed conics, we next associate in an analogous manner a second intrinsic conic system to a strictly convex curve $\gamma$. For this purpose it will be more convenient to assume that we are in $P^{2}$, since otherwise we would for example need to take both branches of a hyperbola into account when defining an interior domain. A conic $C$ in $P^{2}$ bounds a 
closed disk $D_{C}$ and we say that it circumscribes a simple closed curve $\gamma$ if $\gamma \subset D_{\gamma}$. It is clear what we mean by a minimal circumscribed conic.

Let $p, q$ be two different points on $\gamma$. Let $\Gamma_{p, q}$ be the one dimensional family of conics that is tangential to $\gamma$ in $p$ and $q$. In one direction, this family converges to the union of the tangent lines of $\gamma$ at $p$ and $q$. Since $\gamma$ is strictly convex, we have circumscribed conics in the family. (Working in an affine plane, we might not have a circumscribed ellipse in this family. This happens e.g. in points where the affine curvature is nonpositive.) Thus there is a unique minimal circumscribed conic in the family $\Gamma_{p, q}$ that we will denote by $C_{p, q}^{\circ}$.

We now define $C_{p, q}^{\circ}$ in the case that $p$ and $q$ coincide. For $p$ on $\gamma$ we let $\Gamma_{p, p}$ be the one dimensional family of conics that is tangential to $\gamma$ in $p$ with multiplicity at least four. We have circumscribed conics in the family since $\gamma$ is strictly convex. There is therefore a unique minimal circumscribed conic in the family $\Gamma_{p, p}$ that we denote by $C_{p, p}^{\circ}$.

Now for any pair of points $(p, q) \in S^{1} \times S^{1}=\gamma \times \gamma$, we define $f_{p, q}^{\circ}$ : $S^{1} \rightarrow 2 \mathbf{N}_{0} \cup\{\infty\}$ to be the function that associates to a point $r \in S^{1}=\gamma$ the multiplicity with which $C_{p, q}^{\circ}$ and $\gamma$ meet in $r$.

We have already seen that $\left\{f_{p, q}^{\bullet}\right\}$ is an intrinsic conic system. The same arguments imply that $\left\{f_{p, q}^{\circ}\right\}$ is an intrinsic conic system. Thus we have the following proposition.

Proposition 4.6. Both $\left\{f_{p, q}^{\bullet}\right\}$ and $\left\{f_{p, q}^{\circ}\right\}$ are intrinsic conic systems on $S^{1}$.

We can now give a proof of Theorem 4.1 .

Proof of Theorem 4.1. The claim is an immediate consequence of the last proposition and Theorem 3.13, as well as the definition of the relevant intrinsic conic systems with help of inscribed and circumscribed conics.

We remind the reader that a sextactic point $p$ on a simple closed curve $\gamma$ is called a clean maximal (resp. minimal) sextactic point if $C_{p, p}^{\bullet} \cap \gamma\left(S^{1}\right)$ (resp. $C_{p, p}^{\circ} \cap \gamma\left(S^{1}\right)$ ) is connected. The following theorem will be proved in [TU2].

THEOREM 4.7. Let $\gamma: S^{1} \rightarrow A^{2}$ be a strictly convex curve. Then $\gamma$ has at least three clean maximal and at least three clean minimal sextactic points. 
In the following we will prove Lemma 2.3 only assuming $C^{4}$-differentiability of the arc $\gamma$. This together with Remark (ii) after Proposition 5.1 should make it clear how to prove the theorems in the introduction for $C^{4}$-curves.

Proof of Lemma 2.3. We assume here that $\gamma:[0,1] \rightarrow P^{2}$ is a simple $C^{4}$-arc.

We assume that $\gamma$ is free of inflection points. Instead of assuming that $\gamma$ is free of sextactic points, we make the following weaker assumptions: We assume that the osculating conic $C_{t}$ at $\gamma(t)$ crosses $\gamma$ in $\gamma(t)$ for every $t \in(0,1)$. We also assume that $\gamma$ enters the interior domain of $C_{0}$ in $\gamma(0)$ and that it lies locally outside of $C_{1}$ in $\gamma(1)$. The claim of the lemma follows if we can show that $C_{0}$ does not meet $C_{1}$. We therefore assume that $C_{0}$ and $C_{1}$ meet. If $\left.\gamma\right|_{(0,1]}$ meets $C_{0}$, then we let $c \in(0,1]$ be the smallest number such that $\gamma(c) \in C_{0}$, and we set $\hat{\gamma}=\left.\gamma\right|_{[0, c]}$. If $\left.\gamma\right|_{(0,1]}$ does not meet $C_{0}$, then we extend the arc $\gamma$ by continuing on $C_{1}$ up to the first point where $C_{1}$ meets $C_{0}$. We denote the extended arc by $\hat{\gamma}$ and assume it to be parameterized on the interval $[0, c]$. We can assume that $\hat{\gamma}$ is a $C^{4}$-regular curve. Now we set

$$
\sigma=\left.\hat{\gamma} \cup C_{0}\right|_{[\hat{\gamma}(0), \hat{\gamma}(c)]} .
$$

and assume that $\sigma$ is parameterized on $[0,1]$ with $\sigma(0)=\sigma(1)=\hat{\gamma}(c)$. Notice that $\sigma$ is simple and can be assumed to be a $C^{4}$-regular arc that makes a loop which possibly does not close smoothly in $\sigma(0)=\sigma(1)$. Notice that the interior angle in $\sigma(0)=\sigma(1)$ is less than or equal to $\pi$. Such a curve $\sigma$ cannot exist because of the following lemma.

LEMMA 4.8. Let $\sigma:[0,1] \rightarrow A^{2}$ be a strictly convex simple closed curve in the affine plane $A^{2}$ that is $C^{4}$-regular everywhere in $[0,1]$, but possibly so that $\dot{\sigma}(0) \neq \dot{\sigma}(1)$. Denote by $D$ the closed domain bounded by $\sigma$. Set $p=\sigma(0)=\sigma(1)$ and suppose that the interior angle $\theta$ at $p$ is less than or equal to $\pi$. Suppose moreover that the affine curvature function $\kappa$ of $\sigma$ is non-decreasing and non-constant. Then there exists a sextactic point $s \in(0,1)$ such that the osculating conic at $s$ does not coincide with the osculating conics at $\sigma(0)$ and $\sigma(1)$.

Proof. First notice that the osculating conic $C_{0}$ at $\gamma(0)$ is not inscribed in $D$ since we are assuming that the curve $\sigma$ has non-decresing and nonconstant affine curvature function and hence there exists a point $\varepsilon \geq 0$ such 
that the closed arc $\sigma([0, \varepsilon])$ (or point if $\varepsilon=0$ ) is a connected component of $C_{0} \cap \sigma$ and $\sigma$ enters the interior domain $D$ of $C_{0}$ in $\sigma(\varepsilon)$.

For each $s, t \in(0,1)$, let $C_{s, t}^{\bullet}$ be the maximal conic inscribed in $D$ with $\sigma(s), \sigma(t) \in C_{s, t}^{\bullet}$ and $C_{s, t}^{\bullet}$ meeting $\sigma$ in $\sigma(s)$ with multiplicity four if $s=t$. Now for any pair of points $(s, t) \in(0,1) \times(0,1)$, we define $f_{s, t}^{\bullet}: S^{1} \rightarrow 2 \mathbf{N}_{0} \cup\{\infty\}$ as follows: We set $f_{s, t}^{\bullet}(p)=2$ if $p$ is on $C_{s, t}^{\bullet}$, otherwise we set $f_{s, t}^{\bullet}(p)=0$. (Notice that $C_{s, t}^{\bullet}$ and $\sigma$ might meet in $p$ if $\theta=0$.) For $r \in S^{1} \backslash\{p\}=\partial D \backslash\{p\}, f_{s, t}^{\bullet}$ is the multiplicity with which $C_{s, t}^{\bullet}$ and $\sigma$ meet in $r$ if it is less than five, otherwise we set $f_{s, t}^{\bullet}(r)=\infty$. Then $f_{s, t}^{\bullet}$ satisfies the axioms of an intrinsic conic system on every closed interval $[a, b]$ such that $0<a<b<1$.

Assume that $\theta<\pi$. We fix two distinct points $t_{0}, s_{0} \in(0,1)$. We set $C=C_{t_{0}, s_{0}}^{\bullet}$. Then $C$ meets $\left.\sigma\right|_{(0,1)}$ with total multiplicity six. Applying Proposition 3.12, we find a point $u \in(0,1)$ such that $F_{t_{0}, u}$ consists of two components one of which is $t_{0}$. By Proposition 3.11, we find a sextactic point $s$ between $t_{0}$ and $u$ whose osculating conic $C_{s}$ is inscribed. Since the interior angle is less than $\pi$ by assumption, $C_{s}$ cannot pass through $p$. This implies $C_{s} \neq C_{1}, C_{0}$.

Next we consider the case $\theta=\pi$. We set

$$
\delta=\inf \left\{t \in(0,1] \mid \sigma(t) \in C_{1}\right\}
$$

If $\delta=0$, there is a sequence $\left(u_{n}\right)_{n \in N}$ converging to zero such that $\sigma\left(u_{n}\right) \in$ $C_{1}$, and hence $C_{0}=C_{1}$, a contradiction. So $\delta>0$.

We fix two distinct points $t_{0}, s_{0} \in(0,1)$ such that $t_{0}<s_{0}$ and $t_{0} \in(0, \delta)$. We set $C=C_{t_{0}, s_{0}}^{\bullet}$.

First we consider the case that $C$ meets $\left.\sigma\right|_{(0,1)}$ with total multiplicity six. We set

$$
t_{0}^{*}=\inf \left\{t \in(0,1] \mid \sigma(t) \in C=C_{t_{0}, s_{0}}^{\bullet}\right\} .
$$

Applying Proposition 3.8 and Lemma 3.1 to the intrinsic circle system $\left(\left(F^{\bullet}\right)_{t_{0}^{*}, p}^{*}\right)_{p \in S^{1}}$, we find a point $u \in(0,1)$ such that $C_{t_{0}^{*}, u}=C_{u, u}^{\bullet}$. By Proposition 3.11, we find a sextactic point $s$ between $t_{0}^{*}$ and $u$ whose osculating conic $C_{s}$ is inscribed. Since $C_{0}$ is not inscribed in $D, C_{s} \neq C_{0}$ is obvious. If $C_{1}$ is not inscribed, $C_{s} \neq C_{1}$ also holds. So we may assume $C_{1}$ is inscribed. Suppose $\kappa(u)=\kappa(1)$. Then $C_{u, u}^{\bullet}=C_{u}=C_{1}$ holds, where $C_{u}$ is the osculating conic at $u$. Since $C_{t_{0}^{*}, u}=C_{u, u}^{\bullet}$, we have $C_{t_{0}^{*}, u}=C_{1}$, which contradics the fact that $C_{1}$ does not pass through $\sigma\left(t_{0}^{*}\right)$. So we have $\kappa(u)<\kappa(1)$ and hence $\kappa(s)<\kappa(1)$, which implies that $C_{s} \neq C_{1}$. 
Finally, we consider the case that $C$ meets $\left.\sigma\right|_{(0,1)}$ only at $t_{0}$ and $s_{0}$ and that the multiplicity is equal to two in both points. Then $C$ must also be tangent to $\sigma$ at the point $p$. We fix two points $t_{1} \in\left(0, t_{0}\right), s_{1} \in\left(t_{0}, s_{0}\right)$, and set $C^{\prime}=C_{t_{1}, s_{1}}^{\bullet}$. If $C^{\prime}$ passes through $p$, then $C^{\prime}$ meets $C$ with total multiplicity five at least and we have $C^{\prime}=C$, which is a contradiction. So $C^{\prime}$ does not pass through $p$. Thus $C^{\prime}$ meets $\left.\sigma\right|_{(0,1)}$ with total multiplicity six. We can now apply the arguments in the last paragraph to $C^{\prime}$ instead of $C$ to find a sextactic point $s$ whose osculating conic is inscribed and different from $C_{0}$ and $C_{1}$.

\section{$\S 5$. An application to simple closed curves}

In this section we prove the theorems in the introduction except Part (i) of Theorem 1.2 which is Mukhopadhyaya's Theorem that we already proved in the last section. Theorem 1.1 is the same as 5.2, Theorem 1.2 (ii) is in 5.3 and 5.5, (iii) is in 5.4, Theorem 1.3 is in 5.4 and 5.5.

We start with a proposition that will be our main technical tool.

Proposition 5.1. Let $\sigma:[0,1] \rightarrow P^{2}$ be an arc of a curve $\hat{\sigma}:[-\epsilon, 1+$ $\epsilon] \rightarrow P^{2}$ for $\epsilon>0$ that does not have any self-intersections. We assume that $\sigma(t)$ is not an inflection point for any $t \in(0,1)$ and that $\sigma(0)$ and $\sigma(1)$ are either inflection or minimal sextactic points of $\hat{\sigma}$. Then there exists a sextactic point on $\sigma \mid(0,1)$.

Remark. (i) One sees from the proof below that there is a maximal sextactic point on $\sigma \mid(0,1)$ if both $\sigma(0)$ and $\sigma(1)$ are inflection points.

(ii) In the proof of Case (b) in the proof below we will be dealing with a curve that is only $C^{5}$ at one point and otherwise smooth. One can see directly that Axiom (A6) is satisfied at this point. Notice that one can define an intrinsic conic system for a strictly convex curves $\gamma$ that is only $C^{4}$ as follows. In the notation of Section 4 one sets

$$
f_{p, q}^{\bullet}(r)= \begin{cases}2 & \text { if } C_{p, q}^{\bullet} \text { meets } \gamma \text { at } r \text { with multiplicity } 2, \\ 4 & \text { if } C_{p, q}^{\bullet} \text { meets } \gamma \text { at } r \text { with multiplicity } 4 \\ \infty & \text { if } C_{p, q}^{\bullet} \text { meets } \gamma \text { at } r \text { with multiplicity higher than four. }\end{cases}
$$

Then $\left\{f_{p, q}^{\bullet}(r)\right\}$ satisfies the axioms of an intrinsic conic system.

(iii) We explain here how one can easily prove a weak version of Proposition 5.1 under generic assumptions on the arc $\sigma$ using affine curvature. Assume that $\sigma:[0,1] \rightarrow A^{2}$ is a regular arc with no inflection points in 
$\sigma(0,1)$ and that the endpoints $\sigma(0)$ and $\sigma(1)$ are inflection points with the property that the tangent lines there only meet $\sigma$ with finite multiplicity. Then we will show below that the open arc $\sigma(0,1)$ contains a sextactic point. Fabricius-Bjerre $[\mathrm{Fa}]$ makes this observation under the stronger assumption that $\sigma$ meets the tangent lines in the endpoints with multiplicity three precisely and uses it to prove a weak version of Theorem 1.1.

To prove the claim in the previous paragraph, we choose coordinates $(x, y)$ in $A^{2}$ such that $\sigma(0)$ corresponds to $(0,0)$ and the $x$-axis is the oriented tangent line of $\sigma$ at $t=0$. After reparameterizing $\sigma$ we can write it as a graph $y=y(x)$ for $x \geq 0$. Since $\sigma$ is of finite type, we have that

$$
y(x)=\alpha x^{n}+o\left(x^{n}\right) .
$$

We can assume that $\alpha>0$ by either changing the orientation of $\sigma$ or the sign of the $y$-coordinate. The affine curvature $\mu(x)$ can be expressed as

$$
\mu(x)=-\frac{1}{9\left(y^{\prime \prime}\right)^{8 / 3}}\left(5\left(y^{\prime \prime \prime}\right)^{2}-3 y^{\prime \prime \prime \prime} y^{\prime \prime}\right),
$$

see $[\mathrm{Bl} 2$, p. 14, formula (83)]. A short calculation shows that

$$
\lim _{t \rightarrow 0+} \mu(t)=-\infty
$$

Similarly it follows that

$$
\lim _{t \rightarrow 1-} \mu(t)=-\infty
$$

As a consequence there is a point on $\sigma(0,1)$ where the affine curvature takes on its maximum value and this point is then the sextactic point whose existence we wanted to show.

Proof of Proposition 5.1. Assume that both $\sigma(0)$ and $\sigma(1)$ are minimal sextactic points. If there is no sextactic point on $\sigma \mid(0,1)$, then by Lemma 2.3 all osculating conics along $\sigma \mid(0,1)$ are disjoint and it follows that the osculating conic at $\sigma(0)$ must contain $\sigma \mid[0,1]$ in its interior domain since $\sigma(0)$ is a minimal sextactic point. In particular, the osculating conic at $\sigma(1)$ is contained in the interior domain of the osculating conic at $\sigma(0)$. We can reverse the roles of $\sigma(0)$ and $\sigma(1)$ in this argument and prove that the osculating conic at $\sigma(0)$ is contained in the interior domain of the osculating conic at $\sigma(1)$ which is a contradiction. Hence the proposition is proved if both $\sigma(0)$ and $\sigma(1)$ are minimal sextactic points. We will therefore assume 
in the rest of the proof that at least one of the points $\sigma(0)$ or $\sigma(1)$ is an inflection point.

In the rest of the proof we will denote the tangent line of $\hat{\sigma}$ in $\sigma(0)$ by $L_{0}$ and the one in $\sigma_{1}(1)$ by $L_{1}$. We will assume $L_{0}$ and $L_{1}$ parameterized such that the tangents of $L_{0}$ and $\sigma$ coincide in $\sigma(0)$ as well as those of $L_{1}$ and $\sigma$ in $\sigma(1)$.

The following three cases can occur, see Figure 5.1.

(a) The curve $\sigma((0,1))$ neither meets $L_{0}$ nor $L_{1}$.

(b) The curve $\sigma((0,1))$ intersects $L_{0}$.

(c) The curve $\sigma((0,1))$ intersects $L_{1}$.

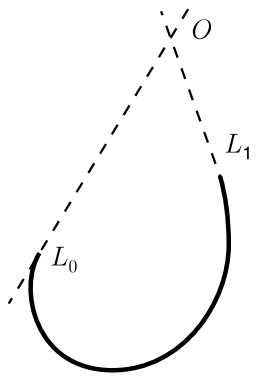

(a)

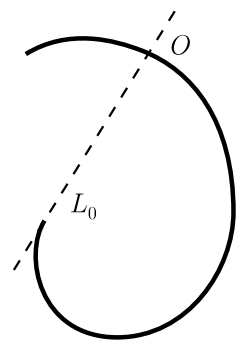

(b)

Figure 5.1

Notice that Case (b) and Case (c) are up to orientation of the curve identical.

(Case (a)) The tangent line $L_{0}$ at $\sigma(0)$ and the tangent line $L_{1}$ at $\sigma(1)$ meet in a point that we denote by $O$. (See Figure 5.1.)

Consider the simple closed curve

$$
\gamma:=\left.\left.L_{0}\right|_{[O, \sigma(0)]} \cup \sigma([0,1]) \cup L_{1}\right|_{[\sigma(1), O]} .
$$

We will first prove that the curve $\gamma$ is nullhomotopic. It follows from Theorem 2.2 that there is an affine plane $A^{2}$ that contains $\sigma([0,1]$ ) (but not necessarily $\gamma)$. Let $L$ be the line segment in this affine plane between $\sigma(0)$ and $\sigma(1)$. The triangle $\left.\left.L_{0}\right|_{[O, \sigma(0)]} \cup L \cup L_{1}\right|_{[\sigma(1), O]}$ is contractible. Hence $\gamma$ is homotopic to $\sigma([0,1]) \cup L$ which is nullhomotopic since it is contained in an affine plane.

Let $D_{\gamma}$ denote the closed disk bounded by $\gamma$. Notice that $L_{0}$ and $L_{1}$ do not meet any interior point of $D_{\gamma}$. One can move $L_{0}\left(\right.$ or $\left.L_{1}\right)$ slightly so 
that it does not meet $D_{\gamma}$. It follows that $\gamma$ and $D_{\gamma}$ lie in an affine plane $A^{2}$ and that $D_{\gamma}$ is convex.

We first assume that both $\sigma(0)$ and $\sigma(1)$ are inflection points. As in Section 4 , we consider inscribed conics. Let $(p, q)$ be a pair of different points on $\sigma([0,1])$. We let $C_{p, q}^{\bullet}$ denote the maximal inscribed conic that lies in $D_{\gamma}$ and is tangential to $\gamma$ in $p$ and $q$. If $p \in \sigma((0,1))$, then $p$ is not an inflection point of $\sigma$ and we can define $C_{p, p}^{\bullet}$ as the maximal inscribed conic that meets $\sigma$ with multiplicity at least four in $p$. We define $f_{p, q}^{\bullet}(r)$ for $r \in \sigma$ to be the multiplicity with which $\hat{\sigma}$ and $C_{p, q}^{\bullet}$ meet. If $r$ lies on the open segments between $O$ and either $\sigma(0)$ or $\sigma(1)$, then we set $f_{p, q}^{\bullet}(r)=2$ if $r \in C_{p, q}^{\bullet}$, otherwise we set $f_{p, q}(r)=0$. One can prove exactly as in the last section that $\left\{f_{p, q}\right\}$ is an intrinsic conic system on the open interval $\sigma((0,1))$. In fact (A8) follows from the fact that $C_{\sigma(0), p}^{\bullet}$ (resp. $\left.C_{p, \sigma(1)}^{\bullet}\right)$ never touches $L_{0} \backslash\{\sigma(0)\}$ (resp. $\left.L_{1} \backslash\{\sigma(1)\}\right)$.

We will now show that $C_{\sigma(0), \sigma(1)}^{\bullet}$ meets $\sigma((0,1))$ in a point $r$. After having shown this the claim of the proposition follows from 3.12 and 3.11 in the case we are now considering.

Assume that there is no such point $r$, i.e., $C_{\sigma(0), \sigma(1)}^{\bullet}$ only meets $\gamma$ in $\sigma(0)$ and $\sigma(1)$. Since $\sigma(0)$ and $\sigma(1)$ are inflection points we have that $C_{\sigma(0), \sigma(1)}^{\bullet}$ and $\hat{\sigma}$ can only meet with multiplicity two both in $\sigma(0)$ and $\sigma(1)$. Of course $C_{\sigma(0), \sigma(1)}^{\bullet}$ also meets $L_{0}$ and $L_{1}$ with multiplicity two in $\sigma(0)$ and $\sigma(1)$. Hence we can increase the conic $C_{\sigma(0), \sigma(1)}^{\bullet}$ in such a way that locally around $\sigma(0)$ and $\sigma(1)$ it stays inside of $D_{\gamma}$. Since $C_{\sigma(0), \sigma(1)}^{\bullet}$ only meets $\gamma$ in $\sigma(0)$ and $\sigma(1)$ this is not only true locally around $\sigma(0)$ and $\sigma(1)$, but globally, contradicting the maximality of $C_{\sigma(0), \sigma(1)}^{\bullet}$. Hence $\gamma$ and $C_{\sigma(0), \sigma(1)}^{\bullet}$ meet in a third point $r$ which must lie on $\sigma((0,1))$ since $C_{\sigma(0), \sigma(1)}^{\bullet}$ can not meet the tangent lines $L_{0}$ and $L_{1}$ except in $\sigma(0)$ and $\sigma(1)$. This finishes the proof when both $\sigma(0)$ and $\sigma(1)$ are inflection points.

Now assume that $\sigma(1)$ is minimal sextactic. We can assume that $\sigma(0)$ is an inflection point as pointed out at the beginning of the proof. We assume that there is no sextactic point on $\sigma((0,1))$. This implies that $\sigma((0,1))$ lies in the interior domain of the osculating conic at $\sigma(1)$ that we will denote by $C$. Notice that $L_{1}$ is tangent to $C$ in $\sigma(1)$, but does not meet it otherwise. The conic $C$ enters $D_{\gamma}$ in $\sigma(1)$ and leaves it in a point $O^{\prime}$ on $L_{0}$ that lies between $\sigma(0)$ and $O$. The curve $\left.\sigma([0,1]) \cup C\right|_{\left[\sigma(1), O^{\prime}\right]}$ is $C^{\infty}$-regular except in $\sigma(1)$ where it is $C^{5}$. Furthermore it satisfies the condition in Case (b). We will prove below that the closed curve

$$
\hat{\gamma}:=\left.\left.L_{0}\right|_{\left[O^{\prime}, \sigma(0)\right]} \cup \sigma([0,1]) \cup C\right|_{\left[\sigma(1), O^{\prime}\right]}
$$


has an inscribed osculating conic. Such a conic can only be osculating at points in $\sigma((0,1))$. It now follows that we have a maximal sextactic point on $\sigma((0,1))$ contradicting our assumption. Notice that it does not follow in this case that we have a maximal sextactic point since we only prove its existence assuming that there is no sextactic points in $\sigma((0,1))$.

If $\sigma(0)$ is minimal sextactic, we can of course use the same argument thus finishing the proof of Case (a).

(Case (b)) We can assume that only one of $\sigma(0)$ and $\sigma(1)$ is minimal sextactic as observed at the beginning of the proof. Assume that $\sigma(0)$ is minimal sextactic and that there is no sextactic point on $\sigma((0,1))$. Then $\sigma([0,1])$ would lie in the closed interior of the osculating conic at $\sigma(0)$ and $L_{0}$ could not meet $\sigma((0,1))$ which contradicts that we are in Case (b). We therefore have a sextactic point on $\sigma((0,1))$ if $\sigma(0)$ is minimal sextactic. Hence we can assume that $\sigma(0)$ is an inflection point. Let $O$ be the point where $L_{0}$ meets $\sigma$ for the first time. (See Figure 5.1.) Consider the simple closed curve

$$
\gamma:=\left.\left.L_{0}\right|_{[O, \sigma(0)]} \cup \sigma\right|_{[\sigma(0), O]} \cdot
$$

In the following we need to include the possibility that $\left.\sigma\right|_{[\sigma(0), O]}$ is the $C^{5}$ curve we met in Case (a). Notice that $\gamma$ bounds a closed convex domain that we denote by $D_{\gamma}$. Exactly as in Case (a), we define for a pair of points $(p, q)$ on $\left.\sigma\right|_{[\sigma(0), O]}$ such that $(p, q) \neq(\sigma(0), \sigma(0)), p \neq O$, and $q \neq O$, the maximal inscribed conic $C_{p, q}^{\bullet}$. We also define $f_{p, q}^{\bullet}$ as in Case (a) and prove that $\left\{f_{p, q}^{\bullet}\right\}$ is an intrinsic conic system on any halfopen arc $(\sigma(0), p] \subset(\sigma(0), O)$ of $\gamma$. (Here we have to check Axiom (A6) separately for the point where the curve from Case (a) is only $C^{5}$.) Fix an arbitrary point $p$ on $(\sigma(0), O)$. Consider the conic $C_{\sigma(0), p}^{\bullet}$. Since $\sigma(0)$ is an inflection point and the angle at $O$ is acute, $C_{\sigma(0), p}^{\bullet}$ must by arguments as in the proof of Case (a) either meet a point $r(\neq p)$ on $\sigma$ between $\sigma(0)$ and $O$ or it meets $\sigma$ in $p$ with multiplicity at least four. Now it follows from Propositions 3.12 and 3.11 that there is a maximal sextactic point on the open arc of $\sigma$ between $\sigma(0)$ and $O$. This proves Case (b).

(Case (c)) Same proof as in Case (b).

ThEOREM 5.2. Let $\gamma: S^{1} \rightarrow P^{2}$ be a simple closed curve without selfintersections that is not nullhomotopic. Then $\gamma$ has at least three sextactic points.

Proof. By Theorem 2.1, due to Möbius, there are at least three intervals of true inflection points on $\gamma$. We therefore find three different arcs 
on $\gamma$ whose endpoints are inflection points. Now the claim of the theorem follows immediately from Proposition 5.1.

Proposition 5.3. Let $\gamma: S^{1} \rightarrow P^{2}$ be a simple closed curve that is nullhomotopic and meets every line in $P^{2}$. Then $\gamma$ has at least four sextactic points.

Proof. By Theorem 2.2, the curve $\gamma$ must have at least four intervals of true inflection points, and hence at least four different subarcs whose endpoints are inflection points. The claim now follows from Proposition 5.1.

THEOREM 5.4. Let $\gamma: S^{1} \rightarrow A^{2}$ be a regular closed convex curve. Then it has at least two sextactic points. The total number of inflection and sextactic points is at least four. In particular, a convex curve with one inflection point has at least three sextactic points.

Proof. We proved in Section 3 that $\gamma$ has at least six sextactic points if it has no inflection points, i.e., if $\gamma$ is strictly convex.

If there is more than one interval of inflection points, then we find at least two sextactic points by Proposition 5.1. We have therefore proved the theorem except when the set of inflection points on $\gamma$ is an interval.

Fix two distinct non-inflection points $a$ and $b$ on $\gamma$. Consider the minimal circumscribed conic $C_{a, b}^{\circ}$ that touches $\gamma$ in $a$ and $b$. If $C_{p, q}^{\circ}$ touches $\gamma$ with multiplicity four in either $a$ or $b$, we consider the intrinsic conic system $\left\{f_{p, q}^{\circ}\right\}$ on the closed interval between $a$ and $b$ which does not contain an inflection point. Then by Proposition 3.11, we find a minimal sextactic point between $a$ and $b$. If $C_{a, b}^{\circ}$ meets $\gamma$ both in $a$ and $b$ with multiplicity two, then there must be a third point $c$ on $\gamma$ lying in $C_{a, b}^{\circ}$. This point cannot be an inflection point since $\gamma$ lies inside of $C_{a, b}^{\circ}$ around $c$. After renaming the points $a, b$ and $c$ we can assume that $c$ lies on the open arc between $a$ and $b$ that is free of inflection points. We now consider the intrinsic conic system $\left\{f_{p, q}^{\circ}\right\}$ on the closed interval $[a, b]$ and use Propositions 3.12 and 3.11 to prove the existence of a minimal sextactic point between $a$ and $b$. In both cases we have a minimal sextactic point and a connected set of inflection points on the curve $\gamma$. There are therefore two open intervals on the curve that are bounded by a minimal sextactic point and an inflection point. Now it follows from Proposition 5.1 that we have a sextactic point on each interval. We have hence proved in this case that there are at least 
three sextactic points on $\gamma$ and one inflection point. This finishes the proof of the theorem.

THEOREM 5.5. Let $\gamma: S^{1} \rightarrow A^{2}$ be a regular closed curve that is not convex. Then it has at least three sextactic points. The total number of inflection and sextactic points is at least six. In particular, if $\gamma$ is not convex and has two inflection points, then it has at least four sextactic points.

Proof. The curve $\gamma$ not being convex has true inflection points. If $\gamma$ has at least three intervals of inflection points, then it has at least three sextactic points by Proposition 5.1. So we may assume that $\gamma$ has exactly two intervals of inflection points that divide $\gamma$ into two arcs. Let $\sigma$ denote the boundary of the convex hull of $\gamma$. Since we are assuming that $\gamma$ has exactly two intervals inflection points, $\sigma$ consists of an arc of $\gamma$ and a line segment between two points $a$ and $b$ on $\gamma$. We choose two arbitrary different points $p$ and $q$ on the open arc of $\gamma$ between $a$ and $b$ and consider the minimal circumscribed conic $C_{p, q}^{\circ}$ touching $\sigma$ in $p$ and $q$. Then the conic $C_{p, q}^{\circ}$ will either meet $\gamma$ in $p$ or $q$ with multiplicity four or it will meet $\gamma$ in a third point $r$. When the second case occurs, by replacing $p$ by $r$ if necessary, we may assume that $r$ lies between $p$ and $q$. In both cases minimal circumscribed conics touching $\sigma$ along $\gamma$ gives rise to an intrinsic conic system on a closed interval of $\gamma$ between $p$ and $q$. Then we can deduce the existence of a minimal sextactic point on the arc between $a$ and $b$ using Propositions 3.11 and 3.12. Proposition 5.1 now implies that there are two further sextactic points on the arcs of $\gamma$ between the inflection points and the minimal sextactic point. There is a fourth sextactic point on the arc between the inflection points that lies inside the convex hull of $\sigma$. This finishes the proof of the theorem.

\section{Appendix A: Simple closed curves with few inflection points}

We will use our paper [TU1] to prove the following theorem which is the second part of Theorem 2.2.

TheOREM A.1. A contractible simple closed curve $\gamma: S^{1} \rightarrow P^{2}$ with less than four intervals of true inflection points is contained in an affine plane.

The first part of Theorem 2.2 is a consequence since a regular simple arc $\sigma:[0,1] \rightarrow P^{2}$ such that no $\sigma(t)$ for $t \in(0,1)$ is an inflection point can 
be shown to be a part of a simple closed curve $\gamma$ with at most two inflection points.

Theorem A.1 will immediately follow from a result on curves on $S^{2}$. In fact, the preimage of $\gamma$ under the canonical projection $p: S^{2} \rightarrow P^{2}$ consists of two simple closed curves since $\gamma$ is contractible. Let $\hat{\gamma}$ be one of these curves. Then the other one is $-\hat{\gamma}$.

A point on $\hat{\gamma}$ is called an inflection point if the osculating circle at that point is a great circle. The inflection points on $\gamma$ and $\hat{\gamma}$ correspond since $p$ maps great circles to lines. We can define a true inflection point of $\gamma$ as a point where the geodesic curvature of $\hat{\gamma}$ changes sign. An interval on $\hat{\gamma}$ is said to consists of true inflection points if the geodesic curvature vanishes and changes sign there. Also the true inflection points on $\gamma$ and $\hat{\gamma}$ correspond.

Theorem A.1 now follows immediately from the following theorem after observing that a lift $\hat{\gamma}$ of the curve $\gamma$ cannot contain a great semicircle as a subarc.

THEOREM A.2. Let $\hat{\gamma}$ be a simple closed curve on $S^{2}$ with at most three intervals of true inflection points. Then $\hat{\gamma}$ lies in a closed hemisphere. Moreover, $\hat{\gamma}$ lies in an open hemisphere if and only if it does not contain any great semicircle as a subarc.

Proof. The claim that $\hat{\gamma}$ lies in a closed hemisphere was already proved in Section 2 of [TU1], see also the arguments later in this proof. It is therefore sufficient to show that $\hat{\gamma}$ lies in an open hemisphere if and only if it does not contain any great semicircle as a subarc.

Assume that $\hat{\gamma}$ contains a great semicircle $J$ as a subarc. Then any great circle on $S^{2}$ meets $J$ and hence $\hat{\gamma}$ too. So $\hat{\gamma}$ can not lie in any open hemisphere.

We now prove the converse. Suppose that $\hat{\gamma}$ is a simple closed curve with at most three intervals of true inflection points and does not contain any great semicircle as a subarc. By [TU1], there exist four points $t_{1}<$ $t_{2}<t_{3}<t_{4}$ on $\hat{\gamma}$ such that $t_{1}, t_{3}$ are clean maximal vertices and $t_{2}, t_{4}$ are clean minimal vertices. The osculating planes of $\hat{\gamma}$, considered as a space curve, at these four points bound a simplex $S$ in $\mathbf{R}^{3}$ containing $\hat{\gamma}$, see [TU1]. If the origin of $\mathbf{R}^{3}$ lies in the interior of this simplex, the curve $\hat{\gamma}$ has at least four intervals of true inflection points, see [TU1], which contradicts our assumption. Thus, the origin lies in the boundary of the simplex $S$. 
Hence the origin lies in an osculating plane $P_{j}$ at one of the clean vertices $t_{j}$. We set $C_{j}=P_{j} \cap S^{2}$. Then $C_{j}$ is a great circle which is an osculating circle of $\hat{\gamma}$ at $t_{j}$. The curve $\hat{\gamma}$ lies completely on one side of $P_{j}$ in $\mathbf{R}^{3}$ and hence also on one side of $C_{j}$ on $S^{2}$, which was the proof in [TU1] that $\hat{\gamma}$ lies in a closed hemisphere. Since $t_{j}$ is a clean vertex we have that $C_{j} \cap \hat{\gamma}$ is connected. Now we use that $\hat{\gamma}$ does not contain a great semicircle as a subarc. It follows that $C_{j} \cap \hat{\gamma}$ is a point or a great circle arc whose length is less than $\pi$. Now let $C_{j}^{+}=\left.C_{j}\right|_{(S, N)}$ be an open great semicircle bounded by two points $S, N$ on $C_{j}$ such that $C_{j} \cap \hat{\gamma}$ is contained in $C_{j}^{+}$. Rotate the great circle $C_{j}$ slightly around the axis in $\mathbf{R}^{3}$ passing through $S$ and $N$ away from $\hat{\gamma}$ into a great circle $\widehat{C}$. If the rotation is sufficiently small, the curve $\hat{\gamma}$ does not meet $\widehat{C}$. Hence $\hat{\gamma}$ lies in an open hemisphere, and we have finished the proof.

\section{Appendix B: Examples of curves with few sextactic points}

In this section we give examples of simple closed curves in the affine plane with few sextactic points that show together with the next appendix that the theorems in the introduction are optimal. Two of these examples are due to Izumiya and Sano [IS] who came up with them in their study of affine evolutes of convex curves.

Part (ii) of Theorem 1.2 is optimal by Example B.1. Part (iii) of Theorem 1.2 is optimal by Example B.2. Theorem 1.3 is optimal by Examples B.2 and B.4.

We know from the proofs in Section 5 that a simple closed curve with more than three (intervals) of inflection points has more than three sextactic points. If it has three inflection points, then it has at least three sextactic point. This is optimal by Example B.1. If it is convex and has two inflection points, it has at least two sextactic points. This is optimal by Example B.2. If it is not convex and has two inflection points, we know from Theorem 5.5 that it has at least four sextactic points. This is optimal by Example B.3. If it is convex and has one inflection point, then it has at least three sextactic points by Theorem 5.4. This is optimal by Example B.4.

ExAMPLE B.1. Here we give an example of a simple closed curve in the affine plane that is not convex, has three inflection points (two of which are true inflection points) and only three sextactic points. This shows that part (ii) of Theorem 1.2 is optimal. 
We identify the affine plane with the complex plane and consider the map

$$
z(t)=t+\frac{3 i}{1+t^{2}} \quad \text { for } t \in \mathbf{R} .
$$

We then get our example by setting

$$
\gamma(t)=1 / z(t) \quad \text { for } t \in \mathbf{R} \cup\{\infty\} .
$$

Notice that the curve is regular at $t=\infty$. In Cartesian coordinates the curve can be expressed as

$$
x(t)=\frac{t}{r(t)}, \quad y(t)=\frac{-3}{r(t)\left(1+t^{2}\right)}
$$

where

$$
r(t)=t^{2}+\frac{9}{\left(1+t^{2}\right)^{2}}
$$

There is a sketch of the curve in Figure B.1, where the inflection points and the sextactic points are marked by $I$ and $S$ respectively.

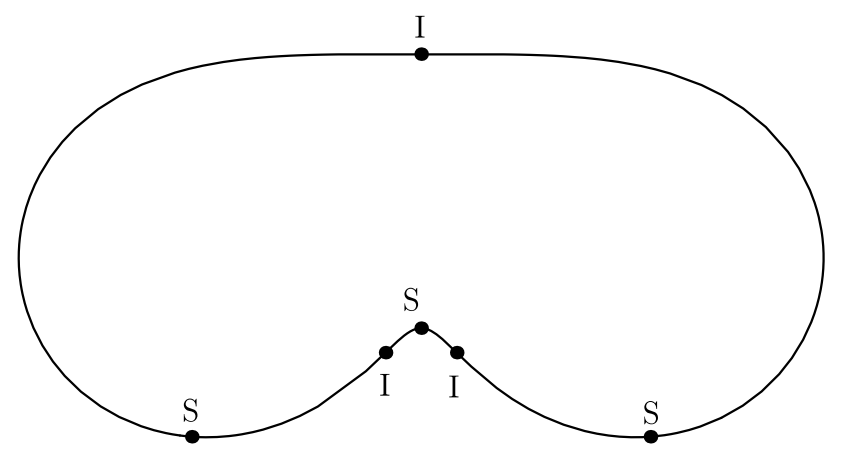

Figure B.1

EXAmple B.2. The following example is due to Izumiya and Sano [IS]. Let the curve $\gamma$ in the affine plane $A^{2}$ be defined in Cartesian coordinates by

$$
x(t)=(\cos (2 t)+5) \cos t, \quad y(t)=(\cos (2 t)+5) \sin t .
$$

This curve is convex and has exactly two sextactic points and two inflection points (which are evidently not true inflection points), showing at the same time that Theorem 1.2 (ii) and Theorem 1.3 are optimal. The affine curvature goes to negative infinity as one approaches the inflection points and has a local maximum between the inflection points. There is a sketch of the curve in Figure B.2. 


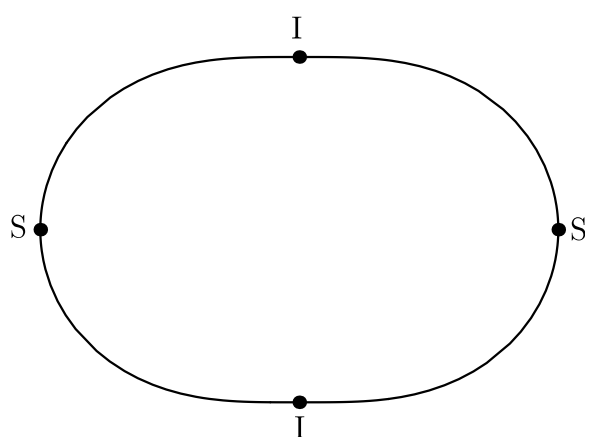

Figure B.2

ExAmple B.3. We consider the curve $\gamma$ in the affine plane $A^{2}$ given in Cartesian coordinates by

$$
x(t)=(3+2 \cos t) \cos t, \quad y(t)=(3+2 \cos t) \sin t .
$$

This curve is not convex. It has two inflection points (both of them true) and four sextactic points. It shows that the last claim in Theorem 5.5 is optimal. There is a sketch of the curve in Figure B.3. Notice that we twice mark $I$ and $S$ at the same place, since the inflection points at $t \approx \pi \pm 0.352$ are so close to the sextactic points at $t \approx \pi \pm 0.335$ that one cannot distinguish between then in the figure.

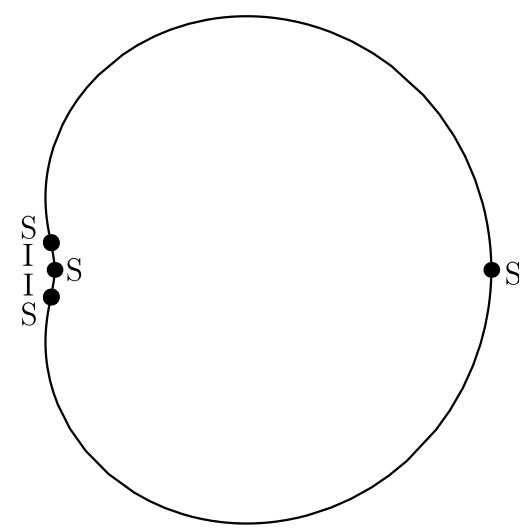

Figure B.3

Example B.4. This example due to Izumiya and Sano [IS] also shows 
that Theorem 1.3 is optimal. Here $\gamma$ is given in Cartesian coordinates by

$$
x(t)=(2+\cos t) \cos t, \quad y(t)=(2+\cos t) \sin t .
$$

The curve is convex. It has one inflection point and three sextactic points. The affine curvature of $\gamma$ has two local maxima, one local minimum and it goes to negative infinity as one approaches the inflection point. This example shows that Theorem 1.3 and the last claim in Theorem 5.4 are optimal. There is a sketch of the curve in Figure B.4.

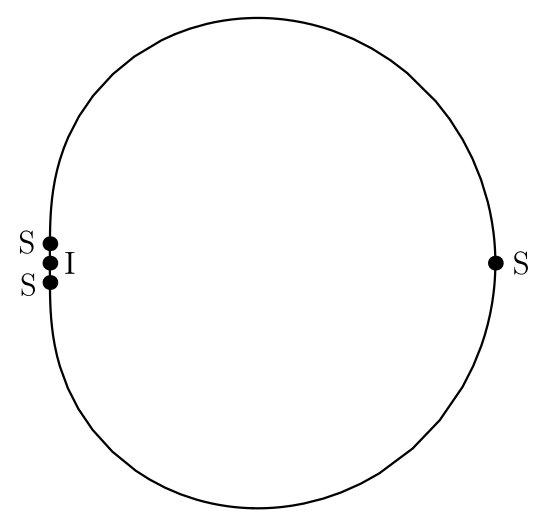

Figure B.4

\section{Appendix C: Sextactic points on a complex plane algebraic curve}

We will give a proof of the theorem of Cayley [Ca2] mentioned in the introduction in this appendix using the theory of inflection points of linear systems as explained in the textbook [Mi], in which one can find explanations of all concepts used here. The proof is essentially only an adaptation of the methods used to prove the formula of Plücker on the number of inflection points in [Mi, p. 241]. We also get a formula for the number of sextactic points when the inflection points are not all simple. This probably also follows from Cayley's method, but we believe that the methods below are simpler. Other proofs of this theorem of Cayley can for example be found in $[\mathrm{Bt}],[\mathrm{Bs}]$, and $[\mathrm{Vi}]$ together with references to further papers on the subject.

We will be considering the linear system of intersection divisors of conics. This linear system corresponds to the Veronese embedding of the curve into $P^{5}(\mathbf{C})$. We will therefore really be studying the number of points of 
higher order contact between a curve in $P^{5}(\mathbf{C})$ and its osculating hyperplanes. Such an approach was used by Barner in [Ba] to prove Mukhopadhyaya's Theorem, see also [Ar3]. As can be seen in these papers, the method can also be used to find the existence of what is called an extatic point of a curve in $P^{2}$, i.e., the analogues of sextactic points when the conics are replaced by algebraic curves of some fixed degree, see [Ar3], but very strong conditions on the curve are needed. The number of extatic points of some given order on an algebraic curve in $P^{2}(\mathbf{C})$ can in principle also be determined as in the following proof.

TheOREM C.1. Let $\gamma$ be a regular algebraic curve of degree $d$ in $P^{2}(\mathbf{C})$. Then $\gamma$ has exactly $3 d(4 d-9)$ sextactic points counted with multiplicities if all inflection points of $\gamma$ are simple. If $\gamma$ has $k$ inflection points with multiplicities $\nu_{1}, \ldots, \nu_{k}$ respectively, then $\gamma$ has

$$
3 d(5 d-11)-\sum_{i=1}^{k} 4 \nu_{i}-3
$$

sextactic points counted with multiplicities.

Proof. Here a conic will not be assumed to be regular. Let $C$ be a conic. Then $C$ induces a divisor $\operatorname{div}(C)$ on $\gamma$ by associating to $p \in \gamma$ the intersection multiplicity of $C$ and $\gamma$ in $p$. By Bézout's theorem, the degree of $\operatorname{div}(C)$ is equal to $2 d$. The collection of these divisors is a complete linear system $Q$ of dimension 5, i.e., $Q$ is a $g_{2 d}^{5}$.

We have to determine the gap numbers for $Q$ at a point $p$. These are the integers $\ell$ at which the dimensions of the spaces in the sequence

$$
Q \supset Q(-p) \supset \cdots \supset Q(-\ell p) \supset \cdots
$$

change. We review that $Q(-\ell p)$ is the space of divisors in $Q$ that meet $\gamma$ in $p$ with multiplicity $\ell$ at least.

Let us first assume that $p$ is not an inflection point and that the multiplicity with which the osculating conic at $p$ meets $\gamma$ in $p$ is $\mu$. Then $\operatorname{dim} Q(-\ell p)=5-\ell$ for $\ell=1,2,3,4,5, \operatorname{dim} Q(-\ell p)=0$ for $\ell=5, \ldots, \mu$ and $Q(-(\mu+1) p)=\emptyset$. Hence the gap sequence is $n_{1}=1, n_{2}=2, n_{3}=3$, $n_{4}=4, n_{5}=5$ and $n_{6}=\mu+1$ if $\mu>5$.

The inflectionary weight of $p$ is by definition equal to

$$
w_{p}(Q)=\sum_{i=1}^{6}\left(n_{i}-i\right) .
$$


Hence $w_{p}(Q)=\mu-5$ if $p$ is not an inflection point. Notice that $\mu-5$ is equal to 0 if $p$ is not a sextactic point. Otherwise $\mu-5$ is the multiplicity of the sextactic point.

Now let us assume that $p$ is an inflection point of $\gamma$ in which the tangent line at $\gamma$ and $\gamma$ meet with multiplicity $\mu$. The dimensions of $Q(-p)$ and $Q(-2)$ do not depend on whether we are at an inflection point or not, i.e., $\operatorname{dim} Q(-p)=4$ and $\operatorname{dim} Q(-2 p)=3$. The spaces $Q(-3 p)=\cdots=Q(-\mu p)$ consist of the divisors of conics that are two lines, one of which is the tangent line, the other one arbitrary. Hence $\operatorname{dim} Q(-\ell p)=2$ for $\ell=3, \ldots, \mu$. The space $Q(-(\mu+1) p)$ consists of the divisors of conics that are two lines, one of which is the tangent line, the other passing through $p$. Hence $\operatorname{dim} Q(-(\mu+1) p)=1$. The spaces $Q(-(\mu+2) p)=\cdots=Q(-2 \mu p)$ consist only of the divisor of the double tangent line. Hence $\operatorname{dim} Q(-(\mu+2) p)=$ $\cdots=\operatorname{dim} Q(-2 \mu p)=0$. The space $Q(-\ell p)=\emptyset$ for $\ell \geq 2 \mu+1$. It follows that $n_{1}=1, n_{2}=2, n_{3}=3, n_{4}=\mu+1, n_{5}=\mu+2, n_{6}=2 \mu+1$. We therefore have $w_{p}(Q)=(\mu-3)+(\mu-3)+(2 \mu-5)=4 \mu-11$. The multiplicity of $p$ as an inflection point is $\nu=\mu-2$. Hence $w_{p}(Q)=4 \nu-3$. If $p$ is a simple inflection point, i.e., $\nu=1$, then $w_{p}(Q)=1$.

We are now going to use the formula

$$
\sum_{p \in \gamma} w_{p}(Q)=6(2 d+5 g-5)
$$

see [Mi, p. 241], where $g$ is the genus of $\gamma$, i.e., $g=(d-1)(d-2) / 2$ by the Plücker formula. Hence the number of sextactic points counted with multiplicities is equal to

$$
3 d(5 d-11)-\sum_{i=1}^{k} 4 \nu_{i}-3 .
$$

If all inflection points are simple, i.e., $\nu_{i}=1$ for all $i$, then the sum is equal to the number of inflection points, which we know to be $3 d(d-2)$. Hence the number of sextactic points is equal to $3 d(4 d-9)$ in that case, and we have finished the proof of the theorem.

ExAMPLE C.2. In this example we will explain the distribution of inflection and sextactic points on regular real and complex cubics. We have referred to the real cubic in this paper as an example for certain of our theorems being optimal. 
We first consider the complex case. Let $\gamma$ be a regular complex plane cubic. First notice that a line and a cubic meet in three points and a conic and cubic in six points. It follows that all inflection and sextactic points on $\gamma$ are simple. We therefore have precisely nine inflection points and precisely twenty seven sextactic points on $\gamma$. The distribution of the inflection points is well known. If we choose one of the inflection points as the origin in the group law of the cubic $\gamma$ and denote it by 0 , then a $p \in \gamma$ is an inflection point if and only if $3 p=0$. Now one can show that all points $p \in \gamma$ with $2 p=0$ are sextactic. These are not all sextactic points. In fact one can show that a point $p \in \gamma$ is either an inflection or a sextactic point if and only if $6 p=0$. Bearing in mind that $\gamma$ as a group is isomorphic to a torus $\mathbf{C} / \Lambda$, we see that the equation $6 p=0$ has thirty six solutions as should be the case.

Now we come to the real parts of regular complex cubics. A real cubic can contain one or two branches. If it consists of one branch, it must correspond to the real part of $\mathbf{C} / \Lambda$ and we see from the above description that it has precisely three inflection points and three sextactic points. If the real cubic consists of two branches, one part must be the real part of $\mathbf{C} / \Lambda$, the other will be the image in $\mathbf{C} / \Lambda$ of the line parallel to the real axis passing through the center of the fundamental domain. Notice that this second branch does not contain any inflection points and is therefore strictly convex. Notice also that it contains precisely six sextactic points.

Acknowledgements. The authors wish to thank S. Izumiya, T. Sano and T. Kurose for fruitful discussions.

\section{REFERENCES}

[Ar1] V. I. Arnold, A ramified covering of $\mathbf{C} P^{2} \rightarrow S^{4}$, hyperbolicity and projective topology, (Russian), Sib. Mat. Zh., 29 (1988), 36-47; English translation in: Sib. Math. J., 29 (1988), 717-726.

[Ar2] V. I. Arnold, Topological Invariants of Plane Curves and Caustics, University Lecture Series 5, American Mathematical Society, Providence, Rhode Island, 1994.

[Ar3] V. I. Arnold, Remarks on the extatic points of plane curves, The Gelfand Mathematical Seminars, 1993-1995, Birkhäuser, Boston (1996), 11-22.

[Ba] M. Barner, Über die Mindestanzahl stationärer Schmiegebenen bei geschlossenen strengkonvexen Raumkurven, Abh. Math. Sem. Univ. Hamburg, 20 (1956), $196-215$.

[Bs] A. B. Basset, On sextactic and allied conics, Quart. J., 46 (1915), 247-252. 
[Bt] G. Battaglini, Sui punti sestatici di una curva qualunque, Atti R. Acc. Lincei, Rend. (Serie quarta) $\mathbf{I V}_{2}$ (1888), 238-246.

[B11] W. Blaschke, Über affine Geometrie VIII: Die Mindestzahl der sextaktischen Punkte einer Eilinie, Leipziger Berichte, 69 (1917), 321-324; Also in: Gesammelte Werke, Band 4, 153-156. Thales Verlag, Essen, 1985.

[Bl2] W. Blaschke, Vorlesungen über Differentialgeometrie II, Affine Differentialgeometrie, Springer-Verlag, Berlin, 1923.

[Bo] G. Bol, Projektive Differentialgeometrie, 1. Teil., Vandenhoeck \& Ruprecht, Göttingen, 1950.

[Ca1] A. Cayley, On the conic of five-pointic contact at any point of a plane curve, Philosophical Transactions of the Royal Society of London CXLIX (1859), 371-400; Also in: The Collected Mathematical Papers, Vol. IV, Cambridge University Press, 1891.

[Ca2] A. Cayley, On the sextactic points of a plane curve, Philosophical Transactions of the Royal Society of London CLV (1865), 548-578; Also in: The Collected Mathematical Papers, Vol. V, Cambridge University Press, 1892.

[Fa] Fr. Fabricius-Bjerre, On a conjecture of G. Bol, Math. Scand., 40 (1977), 194-196.

[GMO] L. Guieu, E. Mourre and V. Yu. Ovsienko, Theorem on six vertices of a plane curve via Sturm theory, The Arnold-Gelfand Mathematical Seminars, Birkhäuser, Boston (1997), 257-266.

[IS] S. Izumiya and T. Sano, Private Communication, 1998.

[Kn] H. Kneser, Neuer Beweis des Vierscheitelsatzes, Christiaan Huygens, 2 (1922/23), 315-318.

[Mi] R. Miranda, Algebraic curves and Riemann surfaces, Graduate Studies in Mathematics 5, American Mathematical Society, Providence, Rhode Island, 1995.

[Mö] A. F. Möbius, Über die Grundformen der Linien der dritten Ordnung, Abhandlungen der Königl. Sächs. Gesellschaft der Wissenschaften, math.-phys. Klasse I (1852), 1-82; Also in: Gesammelte Werke, vol. II, Verlag von S. Hirzel, Leipzig, $1886,89-176$.

[Mu1] S. Mukhopadhyaya, New methods in the geometry of a plane arc, I, Bull. Calcutta Math. Soc., 1 (1909), 31-37; Also in: Collected geometrical papers, vol. I. Calcutta University Press, Calcutta (1929), 13-20.

[Mu2] S. Mukhopadhyaya, Sur les nouvelles méthodes de géometrie, C. R. Séance Soc. Math. France, année 1933 (1934), 41-45.

[TU1] G. Thorbergsson and M. Umehara, A unified approach to the four vertex theorems, II, Differential and symplectic topology of knots and curves (S. Tabachnikov, ed.), Amer. Math. Soc. Transl., Ser. 2, 190 (1999), 229-252.

[TU2] G. Thorbergsson and M. Umehara, On global properties of flexes of periodic functions, preprint (2001).

[Um] M. Umehara, A unified approach to the four vertex theorems, I, Differential and symplectic topology of knots and curves (S. Tabachnikov, ed.), Amer. Math. Soc. Transl., Ser. 2, 190 (1999), 185-228.

[Vi] A. O. Viro, Differential geometry "in the large" of plane algebraic curves, and 
integral formulas for invariants of singularities, (Russian), Zap. Nauchn. Sem. S.-Petersburg. Otdel. Mat. Inst. Steklov. (POMI), 231 (1995), 255-268.

[Wa] C. T. C. Wall, Duality of real projective plane curves: Klein's equation, Topology, 35 (1996), 355-362.

Gudlaugur Thorbergsson

Mathematisches Institut

Universität zu Köln

Weyertal 86-90

50931 Köln

Germany

gthorbergsson@mi.uni-koeln.de

Masaaki Umehara

Department of Mathematics

Graduate School of Science

Hiroshima University

Higashi-Hiroshima, 739-8526

Japan

umehara@math.sci.hiroshima-u.ac.jp 